\title{
SENTRY Antimicrobial Surveillance Program Report: Latin American and Brazilian Results for 1997 through 2001
}

\author{
Helio S. Sader ${ }^{1,2}$, Ronald N. Jones ${ }^{2}$, Ana C. Gales ${ }^{1}$, \\ Juliana B. Silva ${ }^{1}$, Antonio C. Pignatari ${ }^{1}$ and the \\ SENTRY Participants Group (Latin America)
}

\author{
Special Laboratory of Clinical Microbiology ${ }^{l}$, \\ Division of Infectious Diseases, Federal University of \\ São Paulo, São Paulo, SP, Brazil; The JONES Group / \\ JMI Laboratories ${ }^{2}$, North Liberty, Iowa, USA
}

\begin{abstract}
The alarming emergence and spread of antimicrobial resistance among common bacteria threatens the effectiveness of therapy for many infections. Surveillance of antimicrobial resistance is essential to identify the major problems and guide adequate control measures. Several resistance surveillance programs have been implemented in North America and Europe in the last decade; however, very few programs have assessed antimicrobial resistance in Latin American countries. The SENTRY Antimicrobial Surveillance Program was initiated in 1997 and represents the most comprehensive surveillance program in place at the present time worldwide. The SENTRY Program collects consecutive isolates from clinically documented infections in more than 80 medical centers worldwide (10 in Latin America). The isolates are collected according to the type of infection (objectives) and susceptibility tested in a central microbiology laboratory by reference broth microdilution methods according to NCCLS guidelines. The Program also incorporated molecular typing (ribotyping and PFGE) and resistance mechanism analysis of selected isolates. In this report we present a very broad analysis of the data generated by testing almost 20,000 bacterial isolates against more than 30 antimicrobial agents. The susceptibility results $\left(\mathrm{MIC}_{50}\right.$, $\mathrm{MIC}_{90}$ and \% susceptible) are presented in 11 tables according to the organism and site of infection. The data from Brazil, as well as the data from isolates collected in 2001, are analyzed separately. This report allows the evaluation of the activities numerous antimicrobial agents against clinical isolates collected in Latin American countries.
\end{abstract}

Key Words: SENTRY, antimicrobial resistance, nosocomial infection, surveillance, Latin America.

The importance of antimicrobial resistance among nosocomial and community-acquired pathogens is acknowledged worldwide. Well-designed antimicrobial surveillance programs are essential in the fight against these cases of resistance [1]. These programs provide important information on the trend in microbial occurrence in different geographical regions and antimicrobial resistance patterns in nosocomial and

Received on 13 July 2003; revised 29 january 2004.

Address for correspondence: Dr.Helio S. Sader, MD., PhD. The JONES Group / JMI Laboratories. 345 Beaver Kreek Centre, Suite A, North Liberty, Iowa 52317. Phone: 1 (319) 665-3370. Fax: 1 (319) 665-3371.E-mail: helio-sader@jmilabs.com

The Brazilian Journal of Infectious Diseases 2004;8(1):25-79 (C) 2004 by The Brazilian Journal of Infectious Diseases and Contexto Publishing. All rights reserved. community-acquired infections. Such information has the potential to guide therapeutic approaches for serious infections, pending directions from local susceptibility testing, and may have value in the prevention and control of infection. However, in order to be effective, surveillance data should be analyzed, tabulated and rapidly presented to the medical community and infection control workers.

The SENTRY Antimicrobial Surveillance Program (SENTRY) was initiated in 1997 and was designed to monitor the spectrum of microbial pathogens and antimicrobial resistance trends for both nosocomial and community-acquired infections on a global scale, by using validated reference quality identification and susceptibility testing methods in designated central laboratories [1-4]. Rapid communication and dissemination of information 
is a guiding principal of the SENTRY Program. This report is a summary of the data collected in the Latin American region during the first five years of the SENTRY Program (1997 through 2001).

\section{Material and Methods}

\section{Study design}

The SENTRY Program was established to monitor the important pathogens and antimicrobial resistance patterns of nosocomial and community-acquired infections via a broad network of sentinel hospitals distributed by geographic location and size. The collection of isolates was done according to the site of infection and/or type of patient. The types of infections that are monitored include bloodstream infections or true bacteremia (BSI, objective A), communityacquired respiratory tract infections (CARTI, objective B), lower respiratory tract infections in hospitalized patients (LRTI, objective C), skin and soft tissue infections in hospitalized patients (SSTI, objective D), urinary tract infections in hospitalized patients (UTI, objective E), gastroenteritis (objective G), $\beta$-haemolytic streptococci from community-acquired infections (objective $\mathrm{H}$ ), and assessment of pathogens and resistance patterns among infected patients in the intensive care unit (ICU, objective I). Objectives G, H and I were introduced into the program in 2001.

Each participating center contributed results (organism identification, date of isolation, antimicrobial susceptibility profile, etc.) for the first 20 consecutive episodes of BSI per month from January 1997 to December 2001, 100 consecutive episodes of LRTI and CARTI per year from 1997 to 2001, 50 consecutive isolates from SSTI per year from 1997 to 2000, and 50 consecutive isolates from UTI per year from 1997 to 1999 and in 2001, 25 consecutive isolates of organisms considered producers of diarrheal diseases (gastroenteritis) collected in 2001, 25 consecutive $\beta$-haemolytic streptococci isolated from community-acquired infections in 2001, and 50 consecutive and clinically relevant bacterial isolates from patients hospitalized in the ICU in 2001. Just one isolate per patient was included in the study and all isolates were saved on transport swabs and sent to the monitoring center (Iowa, USA) for storage and further characterization by reference identification and susceptibility testing methods. We evaluated all the isolates that were recovered and the data collected from January 1997 to December 2001.

\section{Participating centers}

The laboratories were distributed in various cites of six countries: Sao Paulo, Rio de Janeiro, Florianopolis, Porto Alegre, and Brasilia, Brazil; Buenos Aires and San Isidro, Argentina; Santiago (two centers), Chile; Medellin, Colombia; Mexico City, Mexico; and Montevideo, Uruguay. In 1998, the center located in Montevideo was replaced by a center in Caracas, Venezuela; and in 1999 the Brazilian center located in Rio de Janeiro was replaced by another Brazilian center in Porto Alegre, which is also located in the South region of Brazil. In 2001, the center located in Mendellin, Colombia was replaced by a center located in Brasilia, Brazil. The selection of participant centers was based on the principle that they should be representative in their respective geographic region.

\section{Species identification}

All isolates were identified at the participating institution by the routine methodology in use at each laboratory. On receipt at the monitoring center, isolates were subcultured on blood agar to ensure viability and purity. Species identification was confirmed by standardized methods or performed by using the Vitek System (bioMérieux, Hazelwood, MO) or API (bioMérieux, Hazelwood, MO). Isolates were frozen at $-70^{\circ} \mathrm{C}$ until they were processed.

\section{Susceptibility testing}

Antimicrobial susceptibility testing of isolates was performed by reference broth microdilution methods, 
as described by the National Committee for Clinical Laboratory Standards (NCCLS) [5]. Microdilution trays were purchased from TREK Diagnostics (Cleveland, OH), MicroScan ${ }^{\circledR}$ (West Sacramento, CA), and PML Microbiologicals (Wilsonville, OR). Antimicrobial agents were obtained from their respective manufactures as laboratory grade powder and included cephalosporins (cefepime, cefuroxime, cefotaxime, ceftriaxone, ceftazidime, cefazolin, cefoxitin, cefaclor, and cefixime), penicillins (ampicillin, penicillin, amoxicillin, and oxacillin), $\beta$-lactamase inhibitor combinations (amoxicillin-clavulanate, ticarcillin-clavulanate, and piperacillin-tazobactam), carbapenems (imipenem and meropenem), a monobactam (aztreonam), fluoroquinolones (ciprofloxacin, levofloxacin, gatifloxacin, and garenoxacin [formerly BMS 284756]), aminoglycosides (amikacin, gentamicin, and tobramycin), macrolides (erythromycin, azithromycin, and clarithromycin), glycopeptides (vancomycin and teicoplanin), the oxazolidinone linezolid, the streptogramin quinupristin-dalfopristin, and other drugs, such as clindamycin, chloramphenicol, tetracycline, rifampin, and trimethoprim-sulfamethoxazole. Quality control was performed by testing E. coli American Type Culture Collection (ATCC) 25922, Pseudomonas aeruginosa ATCC 27853, and other strains, as recommended by the NCCLS $[5,6]$. Interpretive criteria for each antimicrobial tested were those published by the NCCLS [5,6].

\section{Results}

Almost 20,000 clinical isolates were analyzed during this period, and the frequencies of the different pathogens were investigated (Table 1). The antimicrobial susceptibility data for the year 2001 were analyzed separately (Tables 2-5), while the data for the entire 5-year period was also compiled (Tables 6-11).

In addition, data collected in the participating Brazilian centers were analyzed separately from the data of the entire Latin American region (including
Brazil). Minimum antimicrobial concentrations that inhibits $50 \%$ and $90 \%$ of the organism collection $\left(\mathrm{MIC}_{50}\right.$ and $\mathrm{MIC}_{90}$, respectively) were presented only for the data of the entire region. Isolates collected from bloodstream infections and from lower respiratory tract infections (mainly pneumonia) in hospitalized patients were also analyzed separately (Tables 3, 5, 7 and 9).

Two groups of pathogens, streptococci and extended-spectrum (ESBL)-producing strains, were analyzed separately due to their importance in community-acquired and nosocomial-acquired infections, respectively. Table 10 presents the antimicrobial susceptibility of streptococcal species, while Table 11 presents the antimicrobial susceptibility of ESBL-producing E. coli and Klebsiella pneumoniae.

\section{Discussion}

We made a comprehensive analysis of the Latin American SENTRY Program data collected during the first five years of this global program. During this period, SENTRY has provided valuable information worldwide [2-4, 7-9]. The rapid publication of regional susceptibility data concerning a large number of antimicrobial agents, indexed by the site of infection, allowed the detection of considerable geographical variation of the in vitro activity of several antimicrobial agents and made evident the emergence of important resistance patterns. These data have been used to guide empirical therapy and the local implementation of control or intervention measures. In addition, SENTRY Program data has guided the implementation of more focused, local surveillance studies, which have been designed based on local resistance problems detected by SENTRY Program results [10,11].

The SENTRY Program has shown that resistance among Gram-negative bacteria seems to be much higher in Latin America when compared to other regions of the world, especially North America and Europe $[1,2]$. On the other hand, some resistance problems related to Gram-positive cocci, such as glycopeptideresistance among enterococci, and high-level penicillin- 


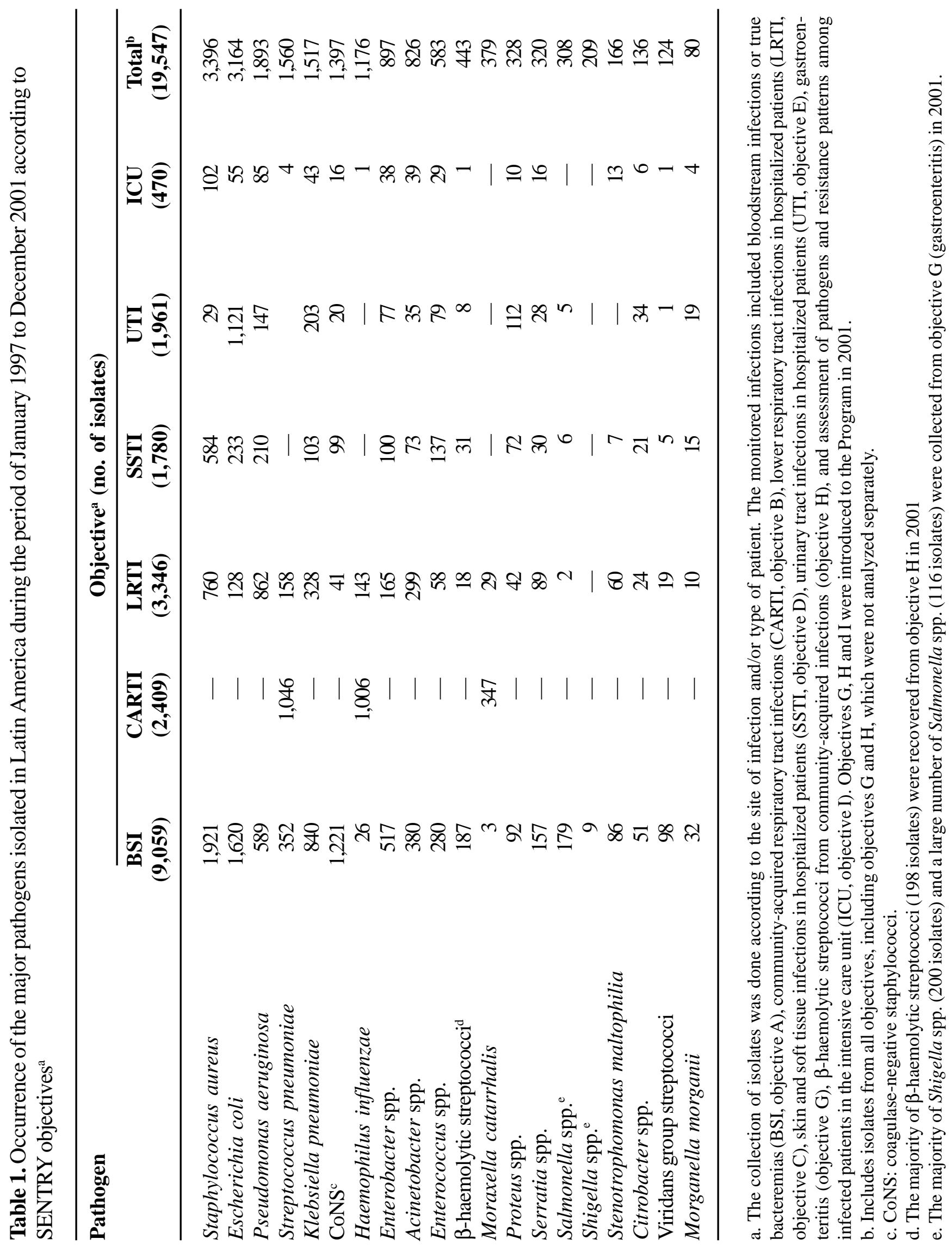


Table 2. Antimicrobial activity and spectrum of drugs tested against the most prevalent Gram-negative pathogens isolated in the year 2001 througout Latin America and in Brazil alone

\begin{tabular}{|c|c|c|c|c|}
\hline \multirow{2}{*}{$\begin{array}{l}\text { Pathogen (no total/Brazil)/ } \\
\text { Antimicrobial agent }\end{array}$} & \multicolumn{2}{|c|}{ Latin America } & \multicolumn{2}{|c|}{ Brazil } \\
\hline & $\mathrm{MIC}_{50}$ & $\mathrm{MIC}_{90}$ & $\%$ Susceptible $^{a}$ & $\%$ Susceptible $^{a}$ \\
\hline \multicolumn{5}{|l|}{ Acinetobacter spp. (166/90) } \\
\hline \multicolumn{5}{|l|}{ Cephalosporins } \\
\hline Ceftazidime & $>16$ & $>16$ & 30.1 & 28.9 \\
\hline Cefepime & $>16$ & $>16$ & 34.3 & 36.7 \\
\hline \multicolumn{5}{|l|}{ Other $\beta$-lactams } \\
\hline Aztreonam & $>16$ & $>16$ & 7.2 & 5.6 \\
\hline Ticarcillin/clavulanate & 128 & $>128$ & 39.8 & 30 \\
\hline Piperacillin/tazobactam & $>64$ & $>64$ & 27.7 & 31.1 \\
\hline Imipenem & 1 & $>8$ & 83.7 & 97.8 \\
\hline Meropenem & 2 & $>8$ & 81.9 & 96.7 \\
\hline \multicolumn{5}{|l|}{ Aminoglycosides } \\
\hline Amikacin & $>32$ & $>32$ & 38 & 35.6 \\
\hline Gentamicin & $>8$ & $>/ 8$ & 30.7 & 38.9 \\
\hline Tobramycin & 16 & $>16$ & 45.8 & 50 \\
\hline \multicolumn{5}{|l|}{ Fluoroquinolones } \\
\hline Ciprofloxacin & $>2$ & $>2$ & 28.3 & 33.3 \\
\hline Levofloxacin & $>4$ & $>4$ & 28.9 & 33.3 \\
\hline Gatifloxacin & $>4$ & $>4$ & 30.1 & 34.4 \\
\hline Garenoxacin & $>4$ & $>4$ & 29.5 & 34.4 \\
\hline \multicolumn{5}{|l|}{ Others } \\
\hline Tetracycline & 8 & $>8$ & 33.7 & 36.7 \\
\hline Trimethoprim/ & & & & \\
\hline sulfamethoxazole & $>2$ & $>2$ & 31.3 & 36.7 \\
\hline Polymyxin B & $\leq 1$ & 2 & 98.2 & - \\
\hline \multicolumn{5}{|l|}{ Enterobacter spp. (186/94) } \\
\hline \multicolumn{5}{|l|}{ Cephalosporins } \\
\hline Cefazolin & $>16$ & $>16$ & 4.3 & 4.3 \\
\hline Cefuroxime & $>16$ & $>16$ & 38.7 & 48.9 \\
\hline Cefoxitin & $>32$ & $>32$ & 2.2 & 2.1 \\
\hline Ceftriaxone & 1 & $>32$ & 61.3 & 73.4 \\
\hline Ceftazidime & $\leq 2$ & $>16$ & 61.8 & 73.4 \\
\hline Cefepime & $\leq 0.12$ & $>16$ & 84.9 & 95.7 \\
\hline \multicolumn{5}{|l|}{ Other $\beta$-lactams } \\
\hline Ampicillin & $>16$ & $>16$ & 2.7 & 3.2 \\
\hline Aztreonam & 0.5 & $>16$ & 60.2 & 72.3 \\
\hline Ticarcillin/clavulanate & 16 & $>128$ & 51.1 & 64.9 \\
\hline Piperacillin/tazobactam & 4 & $>64$ & 65.6 & 76.6 \\
\hline
\end{tabular}




\begin{tabular}{|c|c|c|c|c|}
\hline Imipenem & 0.5 & 1 & 99.5 & 98.9 \\
\hline Meropenem & $\leq 0.06$ & 0.25 & 98.9 & 97.9 \\
\hline \multicolumn{5}{|l|}{ Aminoglycosides } \\
\hline Amikacin & 2 & $>32$ & 84.9 & 91.5 \\
\hline Gentamicin & $\leq 1$ & $>8$ & 67.7 & 81.9 \\
\hline Tobramycin & 0.5 & $>16$ & 62.4 & 78.7 \\
\hline \multicolumn{5}{|l|}{ Fluoroquinolones } \\
\hline Ciprofloxacin & 0.03 & $>2$ & 78 & 89.4 \\
\hline Levofloxacin & 0.03 & $>4$ & 78.5 & 91.5 \\
\hline Gatifloxacin & 0.03 & $>4$ & 79.6 & 92.6 \\
\hline Garenoxacin & 0.12 & $>4$ & 76.9 & 90.4 \\
\hline \multicolumn{5}{|l|}{ Others } \\
\hline Tetracycline & $\leq 4$ & $>8$ & 71.5 & 81.9 \\
\hline $\begin{array}{l}\text { Trimethoprim/ } \\
\text { sulfamethoxazole }\end{array}$ & $<0.5$ & $>2$ & 68.8 & 77.7 \\
\hline Polymyxin B & $\leq 1$ & $>8$ & 79.9 & - \\
\hline \multicolumn{5}{|l|}{ E. coli $(457 / 158)$} \\
\hline \multicolumn{5}{|l|}{ Cephalosporins } \\
\hline Cefazolin & $\leq 2$ & 16 & 85.6 & 89.2 \\
\hline Cefuroxime & 4 & 8 & 90.6 & 93 \\
\hline Cefoxitin & 4 & 8 & 92.8 & 93.7 \\
\hline Ceftriaxone & $\leq 0.25$ & 0.25 & $95.6(5.5)^{\mathrm{b}}$ & $98.1(3.2)^{\mathrm{b}}$ \\
\hline Ceftazidime & $\leq 2$ & $\leq 2$ & $96.1(6.6)^{b}$ & $96.8(3.8)^{b}$ \\
\hline Cefepime & $\leq 0.12$ & $0 . \overline{2} 5$ & 97.2 & 98.1 \\
\hline \multicolumn{5}{|l|}{ Other $\beta$-lactams } \\
\hline Ampicillin & $>16$ & $>16$ & 40.9 & 41.1 \\
\hline Aztreonam & 0.12 & 0.25 & $95.6(5.5)^{\mathrm{b}}$ & $97.5(2.5)^{b}$ \\
\hline Ticarcillin/clavulanate & 8 & 64 & 70.5 & 80.4 \\
\hline Piperacillin/tazobactam & 1 & 4 & 95 & 96.8 \\
\hline Imipenem & 0.12 & 0.12 & 99.8 & 100 \\
\hline Meropenem & $\leq 0.06$ & $\leq 0.06$ & 100 & 100 \\
\hline \multicolumn{5}{|l|}{ Aminoglycosides } \\
\hline Amikacin & 2 & 4 & 97.2 & 100 \\
\hline Gentamicin & $\leq 1$ & 8 & 89.3 & 95.6 \\
\hline Tobramycin & 1 & 8 & 89.7 & 95.6 \\
\hline \multicolumn{5}{|l|}{ Fluoroquinolones } \\
\hline Ciprofloxacin & 0.01 & $>2$ & 85.8 & 91.1 \\
\hline Levofloxacin & 0.03 & 4 & 87.1 & 93 \\
\hline Gatifloxacin & 0.03 & 4 & 87.1 & 92.4 \\
\hline Garenoxacin & 0.03 & $>4$ & 85.8 & 91.1 \\
\hline \multicolumn{5}{|l|}{ Others } \\
\hline $\begin{array}{l}\text { Tetracycline } \\
\text { Trimethoprim/ }\end{array}$ & $\leq 4$ & 8 & 58.4 & 58.2 \\
\hline
\end{tabular}




\begin{tabular}{lcccc}
\hline sulfamethoxazole & $\leq 0.5$ & $>2$ & 52.3 & 44.3 \\
Polymyxin B & $\leq 1$ & $\leq 1$ & 99.4 & -
\end{tabular}

\section{Haemophilus influenzae (319/129)}

Cephalosporins

Cefazolin

Cefuroxime

Cefoxitin

Ceftriaxone

Ceftazidime

Cefepime

Other $\beta$-lactams

Ampicillin

Aztreonam

Piperacillin/tazobactam

Imipenem

Meropenem

Macrolides

Azithromycin

Clarithromycin

Aminoglycosides

Amikacin

Gentamicin

Tobramycin

Fluoroquinolones

Ciprofloxacin

Levofloxacin

Gatifloxacin

Garenoxacin

Others

Tetracycline

Choranphenicol

Trimethoprim/

sulfamethoxazole
8

1

2

0.01

$\leq 2$

0.06

$\leq 0.5$

0.12

$\leq 0.5$

0.25

0.06

1

8

4

2

2

0.03

0.03

0.03

0.03

$\leq 4$

$\leq 2$

$\leq 0.5$
16

2

0.5

$\leq 2$

0.12

$>4$

0.12

$\leq 0.5$

0.5

0.06

\section{2}

8

8

2

4

0.03

0.03

0.03

0.03

$\leq 4$

$\leq 2$

$>2$

54.9

100
100

100

100
100

99.6

93.4

\section{NA}

NA

NA

100

100

100

100

80.3

95.6
NA

96.1

NA

100

100

100

$82.2^{\mathrm{c}}$

100

100

100

100

99.2

91.4

NA

NA

NA

100

100

100

100

93.8

96.1

48.1

\section{K. pneumoniae (292/143)}

Cephalosporins

Cefazolin

Cefuroxime

Cefoxitin

Ceftriaxone

Ceftazidime

Cefepime

Other $\beta$-lactams

$\begin{array}{cr}4 & >16 \\ 4 & >16 \\ 4 & 16 \\ 0.25 & >32 \\ \leq 2 & >16 \\ 0.12 & >16\end{array}$

54.1

46.3

56.5

54.5

83.6

83.9

$65.4(39.7)^{\mathrm{b}}$

$59.4(43.4)^{\mathrm{b}}$

$70.9(37)^{\mathrm{b}}$

77.1

$65.7(42)^{\mathrm{b}}$

70.6 


\begin{tabular}{lcccc}
\hline Ampicillin & $>16$ & $>16$ & 3.8 & 2.8 \\
Aztreonam & 0.12 & $>16$ & $64.7(38)^{\mathrm{b}}$ & $57.3(43.4)^{\mathrm{b}}$ \\
Ticarcillin/clavulanate & 16 & $>128$ & 54.1 & 51.7 \\
Piperacillin/tazobactam & 2 & $>64$ & 76 & 72.7 \\
Imipenem & 0.12 & 0.25 & 100 & 100 \\
Meropenem & $\leq 0.06$ & 0.12 & 99 & 98.6 \\
Aminoglycosides & & & & 86.7 \\
Amikacin & 2 & 32 & 83.6 & 62.9 \\
Gentamicin & $\leq 1$ & $>8$ & 63.7 & 55.9 \\
Tobramycin & 0.5 & $>16$ & 58.6 & 82.5 \\
Fluoroquinolones & & & & 82.5 \\
Ciprofloxacin & 0.03 & $>2$ & 84.2 & 82.5 \\
Levofloxacin & 0.06 & $>4$ & 86 & 83.2 \\
Gatifloxacin & 0.06 & $>4$ & 86 & 64.3 \\
Garenoxacin & 0.12 & $>4$ & 83.9 & \\
Others & & & & 60.1 \\
Tetracycline & $\leq 4$ & $>8$ & 66.8 & \\
Trimethoprim/ & $\leq 2$ & 67.1 & \\
sulfamethoxazole & $\leq 1$ & $\leq 1$ & 97.8 & \\
PolymyxinB & & &
\end{tabular}

\section{Moraxella catarrhalis ${ }^{\mathrm{d}}(63 / 16)$}

$\beta$-lactams

Ampicillin

Cefuroxime

$\leq 2$

1

4

2

$0^{\mathrm{c}}$

Ceftriaxone

0.25

0.5

100

Cefepime

0.5

2

100

Macrolides

Azithromycin

Clarithromycin

\section{$\leq 0.12 \leq 0.12$}

$0^{\mathrm{c}}$

100

100

100

Fluoroquinolones

Ciprofloxacin

$\leq 0.25 \leq 0.25$

100

100

$0.03 \quad 0.03$

100

100

Levofloxacin

0.03

100

100

Gatifloxacin

0.03

0.03

100

100

Garenoxacin

0.03

0.03

100

Others

Tetracycline

Chloramphenicol

$\leq 2 \quad \leq$

$\leq 2$

100

100

Trimethoprim/

sulfamethoxazole

$\leq 0.5 \leq 0.5$

95.2

100

100

100

Proteus spp.(32/-e)

Cephalosporins

Cefazolin

$>16$

62.5

81.3 


\begin{tabular}{|c|c|c|c|c|}
\hline Cefuroxime & 2 & $>16$ & 100 & $\bar{d}$ \\
\hline Cefoxitin & 4 & 4 & 100 & d \\
\hline Ceftriaxone & 0.25 & 32 & 78.1 & d \\
\hline Ceftazidime & $\leq 2$ & $\leq 2$ & 100 & d \\
\hline Cefepime & 0.12 & $>\overline{16}$ & 81.3 & $\mathrm{~d}$ \\
\hline \multicolumn{5}{|l|}{ Other $\beta$-lactams } \\
\hline Ampicillin & $\leq 2$ & $>16$ & 62.5 & d \\
\hline Aztreonam & $\overline{0.12}$ & 1 & 96.9 & d \\
\hline Ticarcillin/clavulanate & $\leq 1$ & 8 & 100 & d \\
\hline Piperacillin/tazobactam & $\leq 0.5$ & 1 & 100 & d \\
\hline Imipenem & 1 & 1 & 100 & d \\
\hline Meropenem & 0.06 & 0.12 & 100 & $\mathrm{~d}$ \\
\hline \multicolumn{5}{|l|}{ Aminoglycosides } \\
\hline Amikacin & 2 & 8 & 96.9 & $\mathrm{~d}$ \\
\hline Gentamicin & $<1$ & $>8$ & 75 & d \\
\hline Tobramycin & 1 & 8 & 81.3 & d \\
\hline \multicolumn{5}{|l|}{ Fluoroquinolones } \\
\hline Ciprofloxacin & 0.03 & $>2$ & 78.1 & d \\
\hline Levofloxacin & 0.06 & $>4$ & 78.1 & d \\
\hline Gatifloxacin & 0.12 & $>4$ & 78.1 & d \\
\hline Garenoxacin & 0.25 & $>4$ & 68.8 & d \\
\hline \multicolumn{5}{|l|}{ Others } \\
\hline Tetracycline & $>8$ & $>8$ & 3.1 & d \\
\hline $\begin{array}{l}\text { Trimethoprim/ } \\
\text { sulfamethoxazole }\end{array}$ & $\leq 0.5$ & $>2$ & 59.4 & d \\
\hline Polymyxin B & $>8$ & $>8$ & 0 & \\
\hline \multicolumn{5}{|l|}{ P. aeruginosa $(407 / 247)$} \\
\hline \multicolumn{5}{|l|}{ Cephalosporins } \\
\hline Ceftazidime & 8 & $>16$ & 56.3 & 50.2 \\
\hline Cefepime & 16 & $>16$ & 54.8 & 46.6 \\
\hline \multicolumn{5}{|l|}{ Other $\beta$-lactams } \\
\hline Aztreonam & 16 & $>16$ & 41.3 & 36 \\
\hline Ticarcillin/clavulanate & 64 & $>128$ & 49.1 & 43.3 \\
\hline Piperacillin/tazobactam & 32 & $>64$ & 64.9 & 56.3 \\
\hline Imipenem & 2 & $>8$ & 62.2 & 51 \\
\hline Meropenem & 2 & $>8$ & 64.4 & 52.6 \\
\hline \multicolumn{5}{|l|}{ Aminoglycosides } \\
\hline Amikacin & 8 & $>32$ & 65.4 & 54.7 \\
\hline Gentamicin & 8 & $>8$ & 49.6 & 42.5 \\
\hline Tobramycin & 1 & $>16$ & 54.5 & 45.7 \\
\hline \multicolumn{5}{|l|}{ Fluoroquinolones } \\
\hline Ciprofloxacin & 1 & $>2$ & 49.9 & 46.2 \\
\hline Levofloxacin & 4 & $>4$ & 49.6 & 46.6 \\
\hline
\end{tabular}




\begin{tabular}{lcccc}
\hline Gatifloxacin & 4 & $>4$ & 46.4 & 43.3 \\
Garenoxacin & $>4$ & $>4$ & 42.8 & 40.9 \\
Others & & & & \\
Tetracycline & $>8$ & $>8$ & 1 & \\
Trimethoprim/ & & & & 5.3 \\
sulfamethoxazole & 2 & $>2$ & 5.4 & \\
Polymyxin B & 2 & 2 & 97 &
\end{tabular}

Salmonella spp. (151/57)

Cephalosporins

Cefazolin

Cefuroxime

$\leq 2 \quad 4$

$4 \quad 8$

96.7

100

Cefoxitin

2

96.7

94.7

Ceftriaxone

0.25

4

97.4

98.2

Ceftazidime

$\leq 2$

0.25

100

100

Cefepime

$\leq 0.12$

$\leq 2$

100

100

Other $\beta$-lactams

Ampicillin

$\leq$

Aztreonam

Ticarcillin/clavulanate

$\leq 0.12$

0.12

100

100

Piperacillin/tazobactam

2

$>16$

86.1

96.5

Imipenem

2

0.12

100

100

90.7

100

99.3

100

0.25

0.25

100

100

Meropenem

$\leq 0.06$

0.06

100

100

Aminoglycosides

Amikacin

12

2

Gentamicin

$\leq 1$

$\leq 2$

100

100

Tobramycin

0.5

96.7

94.6

96.7

94.7

Fluoroquinolones

Ciprofloxacin

0.01

0.12

100

100

Levofloxacin

0.03

0.25

100

100

Gatifloxacin

0.03

0.12

100

100

Garenoxacin

0.06

0.25

100

100

Others

Tetracycline

$\leq 4$

84.1

91.2

Trimethoprim/

sulfamethoxazole

$\leq 0.5 \leq 0.5$

96

96.5

Serratia spp. (66/39)

Cephalosporins

Cefazolin

Cefuroxime

$>16$

$>16$

1.5

0

Cefoxitin

$>16>16$

1.5

0

$32>32$

3

2.6

Ceftriaxone

$0.5>32$

69.7

69.2

Ceftazidime

$\leq 2>16$

83.3

76.9 


\begin{tabular}{|c|c|c|c|c|}
\hline Cefepime & 0.25 & $>16$ & 77.3 & 79.5 \\
\hline \multicolumn{5}{|l|}{ Other $\beta$-lactams } \\
\hline Ampicillin & $>16$ & $>16$ & 1.5 & 0 \\
\hline Aztreonam & 0.5 & $>16$ & 74.2 & 74.4 \\
\hline Ticarcillin/clavulanate & 16 & $>128$ & 50 & 43.6 \\
\hline Piperacillin/tazobactam & 4 & 64 & 71.2 & 61.5 \\
\hline Imipenem & 1 & 2 & 98.5 & 100 \\
\hline Meropenem & 0.06 & 0.25 & 100 & 100 \\
\hline \multicolumn{5}{|l|}{ Aminoglycosides } \\
\hline Amikacin & 4 & $>32$ & 68.2 & 64.1 \\
\hline Gentamicin & $\leq 1$ & $>8$ & 59.1 & 53.8 \\
\hline Tobramycin & 4 & $>16$ & 51.5 & 43.6 \\
\hline \multicolumn{5}{|l|}{ Fluoroquinolones } \\
\hline Ciprofloxacin & 0.12 & $>2$ & 72.7 & 59 \\
\hline Levofloxacin & 0.25 & $>4$ & 77.3 & 64.1 \\
\hline Gatifloxacin & 0.25 & $>4$ & 77.3 & 64.1 \\
\hline Garenoxacin & 2 & $>4$ & 60.6 & 53.8 \\
\hline \multicolumn{5}{|l|}{ Others } \\
\hline Tetracycline & $>8$ & $>8$ & 0 & 0 \\
\hline Trimethoprim/ & & & & \\
\hline sulfamethoxazole & $\leq 0.5$ & $>2$ & 66.7 & 64.1 \\
\hline Polymyxin B & $>8$ & $>8$ & 0 & \\
\hline \multicolumn{5}{|l|}{ Shigella spp. (202/84) } \\
\hline \multicolumn{5}{|l|}{ Cephalosporins } \\
\hline Cefazolin & $\leq 2$ & 4 & 99 & 98.8 \\
\hline Cefuroxime & 2 & 4 & 99.5 & 98.8 \\
\hline Cefoxitin & 2 & 4 & 99.5 & 98.8 \\
\hline Ceftriaxone & $\leq 0.25$ & 0.25 & 100 & 100 \\
\hline Ceftazidime & $\leq 2$ & 2 & 100 & 100 \\
\hline Cefepime & 0.12 & 0.25 & 100 & 100 \\
\hline \multicolumn{5}{|l|}{ Other $\beta$-lactams } \\
\hline Ampicillin & $>16$ & $>16$ & 30.2 & 28.6 \\
\hline Aztreonam & $\leq 0.12$ & 0.12 & 99.5 & 98.8 \\
\hline Ticarcillin/clavulanate & 32 & 32 & 83.7 & 86.9 \\
\hline Piperacillin/tazobactam & 2 & 2 & 100 & 100 \\
\hline Imipenem & 0.12 & 0.25 & 100 & 100 \\
\hline Meropenem & 0.06 & 0.06 & 100 & 100 \\
\hline \multicolumn{5}{|l|}{ Aminoglycosides } \\
\hline Amikacin & 4 & 8 & 100 & 100 \\
\hline Gentamicin & $\leq 1$ & 2 & 100 & 100 \\
\hline Tobramycin & 1 & 2 & 100 & 100 \\
\hline \multicolumn{5}{|l|}{ Fluoroquinolones } \\
\hline Ciprofloxacin & $\leq 0.01$ & $\leq 0.01$ & 100 & 100 \\
\hline
\end{tabular}




\begin{tabular}{|c|c|c|c|c|}
\hline Levofloxacin & $\leq 0.03$ & 0.03 & 100 & 100 \\
\hline Gatifloxacin & $\leq 0.03$ & 0.03 & 100 & 100 \\
\hline Garenoxacin & $\leq 0.03$ & 0.03 & 100 & 100 \\
\hline \multicolumn{5}{|l|}{ Others } \\
\hline Tetracycline & $\leq 4$ & $>8$ & 53.5 & 71.4 \\
\hline $\begin{array}{l}\text { Trimethoprim/ } \\
\text { sulfamethoxazole }\end{array}$ & $>2$ & $>2$ & 26.7 & 15.5 \\
\hline \multicolumn{5}{|c|}{ Stenotrophomonas maltophilia $(\mathbf{7 0} / 27)$} \\
\hline \multicolumn{5}{|l|}{ Cephalosporins } \\
\hline Ceftazidime & 8 & $>16$ & 54.3 & 74.1 \\
\hline Cefepime & 16 & $>16$ & 30 & 44.4 \\
\hline \multicolumn{5}{|l|}{ Other $\beta$-lactams } \\
\hline Aztreonam & $>16$ & $>16$ & 2.9 & 0 \\
\hline Ticarcillin/clavulanate & 32 & 128 & 45.7 & 63 \\
\hline Piperacillin/tazobactam & 64 & $>64$ & 21.7 & 29.6 \\
\hline Imipenem & $>8$ & $>8$ & 0 & 0 \\
\hline Meropenem & $>8$ & $>8$ & 2.9 & 7.4 \\
\hline \multicolumn{5}{|l|}{ Aminoglycosides } \\
\hline Amikacin & $>32$ & $>32$ & 10 & 11.1 \\
\hline Gentamicin & $>8$ & $>8$ & 5.7 & 11.1 \\
\hline Tobramycin & $>16$ & $>16$ & 7.1 & 11.1 \\
\hline \multicolumn{5}{|l|}{ Fluoroquinolones } \\
\hline Ciprofloxacin & 1 & $>2$ & 55.7 & 48.1 \\
\hline Levofloxacin & 0.5 & 1 & 98.6 & 100 \\
\hline Gatifloxacin & 0.5 & 1 & 98.6 & 100 \\
\hline Garenoxacin & 1 & 4 & 88.6 & 81.5 \\
\hline \multicolumn{5}{|l|}{ Others } \\
\hline Tetracycline & $>8$ & $>8$ & 11.4 & 11.1 \\
\hline $\begin{array}{l}\text { Trimethoprim/ } \\
\text { sulfamethoxazole }\end{array}$ & $\leq 0.5$ & $\leq 0.5$ & 98.6 & 100 \\
\hline Polymyxin B & 2 & 8 & 59.2 & - \\
\hline
\end{tabular}

a. Interpreted by NCCLS 2003 criteria, when available [6] except for polymyxin B ( $\leq 2$ for susceptible and $\geq 4$ for resistant);

b. Percentage of strain with MIC $\geq 2 \mu \mathrm{g} / \mathrm{mL}$, indicating possible ESBL production;

c. Percentage of non- $\beta$-lactamase producing strains. This value also represents the susceptibility rate to amoxicillin.

d. Breakpoints for Haemophilus influenzae were used;

e. The results were not analyzed because the number of isolates was low $(<10)$.

NA - Not applicable because there was no breakpoint established by the NCCLS [6]. 
Table 3. Antimicrobial activity and spectrum of drugs tested against the most prevalent Gram-positive cocci isolated from hospitalized patients in 2001

\begin{tabular}{|c|c|c|c|c|}
\hline \multirow{2}{*}{$\begin{array}{l}\text { Pathogen (no total/Brazil)/ } \\
\text { Antimicrobial agent }\end{array}$} & \multicolumn{2}{|c|}{ America } & \multicolumn{2}{|c|}{ Brazil } \\
\hline & $\mathrm{MIC}_{50}$ & $\mathrm{MIC}_{90}$ & $\%$ Susceptible $^{a}$ & $\%$ Susceptible $^{\mathrm{a}}$ \\
\hline \multicolumn{5}{|l|}{ S. aureus $(669 / 329)$} \\
\hline \multicolumn{5}{|l|}{ Cephalosporins } \\
\hline Ceftriaxone & 4 & $>32$ & $56.2^{\mathrm{b}}$ & $56.8^{\mathrm{b}}$ \\
\hline Cefepime & 4 & $>16$ & $56.2^{\mathrm{b}}$ & $56.8^{b}$ \\
\hline \multicolumn{5}{|l|}{ Other $\beta$-lactams } \\
\hline Oxacillin & 0.5 & $>8$ & 56.2 & 56.8 \\
\hline Penicillin & 16 & $>32$ & 7.5 & 8.8 \\
\hline Amoxicillin/clavulanate & $\leq 2$ & $>16$ & $56.2^{\mathrm{b}}$ & $56.8^{b}$ \\
\hline Piperacillin/tazobactam & 2 & $>64$ & $56.2^{\mathrm{b}}$ & $56.8^{b}$ \\
\hline Imipenem & 0.06 & $>8$ & $56.2^{\mathrm{b}}$ & $56.8^{\mathrm{b}}$ \\
\hline \multicolumn{5}{|l|}{ MLS } \\
\hline Clindamycin & 0.12 & $>8$ & 60.5 & 59.9 \\
\hline Erythromycin & 0.5 & $>8$ & 50.7 & 48.9 \\
\hline \multicolumn{5}{|l|}{ Fluoroquinolones } \\
\hline Ciprofloxacin & 0.5 & $>2$ & 58.4 & 59.9 \\
\hline Levofloxacin & 0.25 & 4 & 62.9 & 65 \\
\hline Gatifloxacin & 0.12 & 4 & 89.7 & 86.9 \\
\hline Garenoxacin & 0.03 & 1 & 97.8 & 97.9 \\
\hline \multicolumn{5}{|l|}{ Others } \\
\hline Gentamicin & $\leq 2$ & $>8$ & 55.3 & 56.2 \\
\hline Rifampin & $\leq 0.25$ & 2 & 75.7 & 63.8 \\
\hline Chloramphenicol & 8 & $>16$ & 72.2 & 67.8 \\
\hline Tetracycline & $\leq 4$ & $>8$ & 74.1 & 59.6 \\
\hline Doxycycline & $\leq 0.5$ & $>4$ & 82.4 & 70.8 \\
\hline Trimethoprim/sulfamethoxale & $\leq 0.5$ & $>2$ & 73.4 & 56.2 \\
\hline Vancomycin & 1 & 1 & 100 & 100 \\
\hline Teicoplanin & 1 & 2 & 99.9 & 99.7 \\
\hline Quinupristin/dalfopristin & 0.25 & 0.5 & 100 & 100 \\
\hline Linezolid & 2 & 2 & 100 & 100 \\
\hline \multicolumn{5}{|l|}{ CoNS (246/147) } \\
\hline \multicolumn{5}{|l|}{ Cephalosporins } \\
\hline Ceftriaxone & 16 & $>32$ & $20.7^{b}$ & $17.7^{\mathrm{b}}$ \\
\hline Cefepime & 4 & $>16$ & $20.7^{b}$ & $17.7^{\mathrm{b}}$ \\
\hline \multicolumn{5}{|l|}{ Other $\beta$-lactams } \\
\hline Oxacillin & $>8$ & $>8$ & 20.7 & 17.7 \\
\hline Penicillin & 8 & $>32$ & 11 & 10.2 \\
\hline Amoxicillin/clavulanate & $\leq 2$ & $>16$ & $20.7^{b}$ & $17.7^{\mathrm{b}}$ \\
\hline Piperacillin/tazobactam & 4 & $>64$ & $20.7^{b}$ & $17.7^{\mathrm{b}}$ \\
\hline Imipenem & 0.5 & $>8$ & $20.7^{b}$ & $17.7^{b}$ \\
\hline MLS & & & & \\
\hline
\end{tabular}




\begin{tabular}{|c|c|c|c|c|}
\hline Clindamycin & 0.12 & $>8$ & 53.7 & 46.9 \\
\hline Erythromycin & $>8$ & $>8$ & 28.5 & 27.9 \\
\hline \multicolumn{5}{|l|}{ Fluoroquinolones } \\
\hline Ciprofloxacin & 0.5 & $>2$ & 52.4 & 44.9 \\
\hline Levofloxacin & 0.5 & $>4$ & 63 & 57.1 \\
\hline Gatifloxacin & 0.25 & 2 & 93.1 & 92.5 \\
\hline Garenoxacin & 0.06 & 2 & 95.1 & 94.6 \\
\hline \multicolumn{5}{|l|}{ Others } \\
\hline Gentamicin & 8 & $>8$ & 44.9 & 39.7 \\
\hline Rifampin & 0.25 & $>2$ & 74.8 & 70.7 \\
\hline Chloramphenicol & 8 & $>16$ & 68.3 & 59.2 \\
\hline Tetracycline & $\leq 4$ & $>8$ & 77.6 & 74.1 \\
\hline Doxycycline & $\leq 0.5$ & $>4$ & 88.6 & 87.8 \\
\hline Trimethoprim/sulfamethoxale & 2 & $>2$ & 48.8 & 35.4 \\
\hline Vancomycin & 2 & 2 & 100 & 100 \\
\hline Teicoplanin & 2 & 8 & 92.3 & 91.2 \\
\hline Quinupristin/dalfopristin & 0.25 & 0.5 & 99.2 & 100 \\
\hline Linezolid & 1 & 2 & 100 & 100 \\
\hline \multicolumn{5}{|l|}{ Enterococcus spp. (102 / 52) } \\
\hline \multicolumn{5}{|l|}{$\beta$-lactams } \\
\hline Ampicillin & $\leq 2$ & 8 & 90.2 & 96.2 \\
\hline Penicillin & 4 & 16 & 70.6 & 61.5 \\
\hline Amoxicillin/Clavulanate & $\leq 2$ & 4 & $90.2^{c}$ & $96.2^{\mathrm{c}}$ \\
\hline Piperacillin/Tazobactam & 4 & $>64$ & $90.2^{c}$ & $96.2^{\mathrm{c}}$ \\
\hline \multicolumn{5}{|l|}{ MLS } \\
\hline Erythromycin & $>8$ & $>8$ & 6.9 & 9.6 \\
\hline \multicolumn{5}{|l|}{ Fluoroquinolones } \\
\hline Ciprofloxacin & 2 & $>2$ & 46.1 & 38.5 \\
\hline Levofloxacin & 2 & $>4$ & 54.9 & 46.2 \\
\hline Gatifloxacin & 1 & $>4$ & 54.9 & 46.2 \\
\hline Garenoxacin & 1 & 4 & 92.2 & 59.6 \\
\hline \multicolumn{5}{|l|}{ Others } \\
\hline $\operatorname{Gentamicin}(\mathrm{HL})$ & $\leq 500$ & $>1,000$ & 55.9 & 42.3 \\
\hline Streptomycin (HL) & $\leq 1,000$ & $>2,000$ & 62.7 & 82.7 \\
\hline Rifampin & $>2$ & $>2$ & 22.5 & 15.4 \\
\hline Chloramphenicol & 8 & $>16$ & 57.8 & 42.3 \\
\hline Tetracycline & $>8$ & $>8$ & 29.4 & 30.8 \\
\hline Doxycycline & $>4$ & $>4$ & 37.3 & 36.5 \\
\hline Trimethoprim/sulfamethoxale & $\leq 0.5$ & $>2$ & 73.5 & 61.5 \\
\hline Vancomycin & 1 & 2 & 93.1 & 92.3 \\
\hline Teicoplanin & 0.12 & 0.5 & 94.1 & 92.3 \\
\hline Quinupristin/dalfopristin & 8 & $>8$ & 6.9 & 3.8 \\
\hline Linezolid & 2 & 2 & 100 & 100 \\
\hline
\end{tabular}

a. Interpreted by NCCLS 2003 criteria, when available [6]; b. Susceptibility is predicted by the oxacillin result [6]; c. Susceptibility is predicted by the ampicillin result [6]; CoNS: coagulase-negative staphylococci; HL: High level aminoglycoside resistance screen. 
Table 4. Antimicrobial activity and spectrum of drugs tested against the most prevalent Gram-negative pathogens isolated in the year 2001 throughout Latin America and in Brazil alone, from patients with bacteremia and pneumonia.

\begin{tabular}{|c|c|c|c|c|}
\hline \multirow{3}{*}{$\begin{array}{l}\text { Pathogen/ } \\
\text { Antimicrobial agents }\end{array}$} & \multicolumn{4}{|c|}{$\%$ Susceptible strains (number of isolates tested) } \\
\hline & \multicolumn{2}{|c|}{ Latin America } & \multicolumn{2}{|c|}{ Brazil } \\
\hline & $\begin{array}{c}\text { Bacteremia } \\
(67)\end{array}$ & $\begin{array}{c}\text { Pneumonia } \\
\text { (60) }\end{array}$ & $\begin{array}{c}\text { Bacteremia } \\
(40)\end{array}$ & $\begin{array}{l}\text { Pneumonia } \\
\text { (29) }\end{array}$ \\
\hline \multicolumn{5}{|l|}{ Acinetobacter spp. } \\
\hline Ceftazidime & 44.8 & 21.7 & 47.5 & 13.8 \\
\hline Cefepime & 49.3 & 23.3 & 55 & 17.2 \\
\hline \multicolumn{5}{|l|}{ Other $\beta$-lactams } \\
\hline Aztreonam & 11.9 & 6.7 & 10 & 3.4 \\
\hline Ticarcillin/clavulanate & 38.8 & 11.7 & 47.5 & 10.3 \\
\hline Piperacillin/tazobactam & 44.8 & 15 & 50 & 6.9 \\
\hline Imipenem & 88.1 & 86.7 & 97.5 & 100 \\
\hline Meropenem & 85.1 & 85 & 97.5 & 96.6 \\
\hline \multicolumn{5}{|l|}{ Aminoglycosides } \\
\hline Amikacin & 50.7 & 33.3 & 55 & 17.2 \\
\hline Gentamicin & 46.3 & 21.7 & 57.5 & 24.1 \\
\hline Tobramycin & 58.2 & 38.3 & 70 & 34.5 \\
\hline \multicolumn{5}{|l|}{ Fluoroquinolones } \\
\hline Ciprofloxacin & 44.8 & 18.3 & 52.5 & 13.8 \\
\hline Levofloxacin & 46.3 & 18.3 & 52.5 & 13.8 \\
\hline Gatifloxacin & 46.3 & 20 & 52.5 & 17.2 \\
\hline Garenoxacin & 46.3 & 20 & 52.5 & 17.2 \\
\hline \multicolumn{5}{|l|}{ Others } \\
\hline Tetracycline & 47.8 & 21.7 & 52.5 & 17.2 \\
\hline $\begin{array}{l}\text { Trimethoprim/ } \\
\text { sulfamethoxazole }\end{array}$ & 46.3 & 23.3 & 52.5 & 24.1 \\
\hline Enterobacter spp. & $\begin{array}{c}\text { Bacteremia } \\
\text { (117) }\end{array}$ & $\begin{array}{c}\text { Pneumonia } \\
(31)\end{array}$ & $\begin{array}{c}\text { Bacteremia } \\
(59)\end{array}$ & $\begin{array}{c}\text { Pneumonia } \\
\text { (14) }\end{array}$ \\
\hline \multicolumn{5}{|l|}{ Cephalosporins } \\
\hline Cefazolin & 3.4 & 6.5 & 3.4 & 7.1 \\
\hline Cefuroxime & 37.6 & 41.9 & 50.8 & 42.9 \\
\hline Cefoxitin & 0.9 & 6.5 & 0 & 7.1 \\
\hline Ceftriaxone & 63.2 & 51.6 & 78 & 57.1 \\
\hline Ceftazidime & 61.5 & 61.3 & 72.9 & 57.1 \\
\hline Cefepime & 86.3 & 77.4 & 96.6 & 92.9 \\
\hline \multicolumn{5}{|l|}{ Other $\beta$-lactams } \\
\hline Ampicillin & 3.4 & 3.2 & 3.4 & 7.1 \\
\hline Aztreonam & 62.4 & 51.6 & 72.9 & 57.1 \\
\hline Ticarcillin/clavulanate & 51.3 & 51.6 & 67.8 & 57.1 \\
\hline
\end{tabular}




\begin{tabular}{|c|c|c|c|c|}
\hline Piperacillin/tazobactam & 67.5 & 61.3 & 83.1 & 57.1 \\
\hline Imipenem & 100 & 96.8 & 100 & 92.9 \\
\hline Meropenem & 99.1 & 96.8 & 98.3 & 92.9 \\
\hline \multicolumn{5}{|l|}{ Aminoglycosides } \\
\hline Amikacin & 84.6 & 87.1 & 93.2 & 85.7 \\
\hline Gentamicin & 69.2 & 67.7 & 83.1 & 85.7 \\
\hline Tobramycin & 63.2 & 67.7 & 79.7 & 85.7 \\
\hline \multicolumn{5}{|l|}{ Fluoroquinolones } \\
\hline Ciprofloxacin & 76.9 & 71 & 86.4 & 92.9 \\
\hline Levofloxacin & 78.6 & 71 & 89.8 & 92.9 \\
\hline Gatifloxacin & 79.5 & 71 & 91.5 & 92.9 \\
\hline Garenoxacin & 77.8 & 71 & 88.1 & 92.9 \\
\hline \multicolumn{5}{|l|}{ Others } \\
\hline Tetracycline & 67.5 & 83.9 & 79.7 & 85.7 \\
\hline \multicolumn{5}{|l|}{ Trimethoprim/ } \\
\hline sulfamethoxazole & 65 & 80.6 & 74.6 & 85.7 \\
\hline $\begin{array}{l}\text { E. coli } \\
(381)\end{array}$ & $\begin{array}{c}\text { Bacteremia } \\
\text { (14) }\end{array}$ & $\begin{array}{c}\text { Pneumonia } \\
(122)\end{array}$ & $\begin{array}{c}\text { Bacteremia } \\
\text { (a) }\end{array}$ & Pneumonia \\
\hline \multicolumn{5}{|l|}{ Cephalosporins } \\
\hline Cefazolin & 87.4 & 64.3 & 93.4 & a \\
\hline Cefuroxime & 91.6 & 85.7 & 95.1 & a \\
\hline Cefoxitin & 93.4 & 85.7 & 93.4 & a \\
\hline Ceftriaxone & $96.3(4.7)^{\mathrm{b}}$ & $92.9(7.1)^{\mathrm{b}}$ & $98.4(2.5)^{\mathrm{b}}$ & a \\
\hline Ceftazidime & $96.3(5.8)^{\mathrm{b}}$ & $100(7.1)^{\mathrm{b}}$ & $96.7(3.3)^{\mathrm{b}}$ & a \\
\hline Cefepime & 97.6 & 100 & 98.4 & a \\
\hline \multicolumn{5}{|l|}{ Other $\beta$-lactams } \\
\hline Ampicillin & 41.7 & 28.6 & 45.1 & a \\
\hline Aztreonam & $96.6(4.7)^{\mathrm{b}}$ & $92.3(7.7)^{\mathrm{b}}$ & $98.4(1.6)^{\mathrm{b}}$ & $\mathrm{a}$ \\
\hline Ticarcillin/clavulanate & 71.4 & 64.3 & 82 & a \\
\hline Piperacillin/tazobactam & 95.8 & 85.7 & 96.7 & a \\
\hline Imipenem & 100 & 92.9 & 100 & a \\
\hline Meropenem & 100 & 100 & 100 & a \\
\hline \multicolumn{5}{|l|}{ Aminoglycosides } \\
\hline Amikacin & 96.9 & 92.9 & 100 & a \\
\hline Gentamicin & 90.3 & 85.7 & 95.9 & a \\
\hline Tobramycin & 90.3 & 92.9 & 95.9 & a \\
\hline \multicolumn{5}{|l|}{ Fluoroquinolones } \\
\hline Ciprofloxacin & 85.8 & 85.7 & 91.8 & a \\
\hline Levofloxacin & 87.4 & 85.7 & 94.3 & $\mathrm{a}$ \\
\hline Gatifloxacin & 87.1 & 92.9 & 93.4 & a \\
\hline Garenoxacin & 85.8 & 92.9 & 91.8 & a \\
\hline \multicolumn{5}{|l|}{ Others } \\
\hline Tetracycline & 58.8 & 42.9 & 61.5 & a \\
\hline
\end{tabular}


Trimethoprim/ sulfamethoxazole

K. pneumoniae

Cephalosporins

Cefazolin

Cefuroxime

Cefoxitin

Ceftriaxone

Ceftazidime

Cefepime

Other $\beta$-lactams

Ampicillin

Aztreonam

Ticarcillin/clavulanate

Piperacillin/tazobactam

Imipenem

Meropenem

Aminoglycosides

Amikacin

Gentamicin

Tobramycin

Fluoroquinolones

Ciprofloxacin

Levofloxacin

Gatifloxacin

Garenoxacin

Others

Tetracycline

Trimethoprim/

sulfamethoxazole

\section{P. aeruginosa}

Cephalosporins

Ceftazidime

Cefepime

Other $\beta$-lactams

Aztreonam

Ticarcillin/clavulanate

Piperacillin/tazobactam

Imipenem

Meropenem

$$
52.8 \quad 57.1
$$

Bacteremia Pneumonia

(176)

(73)

56.3

58.5

84.7

$66.5(37.5)^{\mathrm{b}}$

$69.9(36.4)^{b}$

79

57.5

58.9

84.9

$69.9(39.7)^{\mathrm{b}}$

$82.2(28.8)^{\mathrm{b}}$

82.2

\section{7}

$64.8(36.9)^{\mathrm{b}}$

56.3

73.9

100

99.4

1.4

$74(32.9)^{\mathrm{b}}$

57.5

84.9

100

100

84.1

63.6

59.7

89

71.2

63

84.7

86.9

90.4

91.8

86.9

91.8

84.1

90.4

64.2

78.1

78.1

65.3

Bacteremia

(119)

Pneumonia

(203)

68.1

66.4

50.7

49.8

47.9

59.7

79.8

79

80.7
39.4

47.8

56.2

53.7

57.3
45.1

Bacteremia Pneumonia

(90)

(28)

$56.7 \quad 53.6$

$60 \quad 53.6$

$86.7 \quad 82.1$

$64.4(37.8)^{\mathrm{b}} \quad 57.1(42.9)^{\mathrm{b}}$

$70(37.8)^{\mathrm{b}} \quad 67.9(39.3)^{\mathrm{b}}$

$75.6 \quad 71.4$

$$
4.4 \quad 0
$$

$62.2(35.6)^{\mathrm{b}} \quad 57.1(42.9)^{\mathrm{b}}$

$55.6 \quad 50$

$74.4 \quad 78.6$

$100 \quad 100$

$98.9 \quad 100$

90

85.7

$65.6 \quad 67.9$

$62.2 \quad 50$

$86.7 \quad 85.7$

$86.7 \quad 85.7$

$\begin{array}{ll}86.7 & 85.7\end{array}$

$86.7 \quad 85.7$

$62.2 \quad 67.9$

$58.9 \quad 71.4$

Bacteremia Pneumonia

(58)

(142)

69

40.8

65.5

38.7

51.7

31

$58.6 \quad 38.7$

$75.9 \quad 46.5$

$75.9 \quad 41.5$

$77.6 \quad 45.1$ 


\begin{tabular}{|c|c|c|c|c|c|}
\hline \multicolumn{6}{|l|}{ Aminoglycosides } \\
\hline Amikacin & 73.9 & 61.1 & 70.7 & & 51.4 \\
\hline Gentamicin & 63 & 43.3 & 69 & & 35.9 \\
\hline Tobramycin & 67.2 & 50.7 & 69 & & 41.5 \\
\hline \multicolumn{6}{|l|}{ Fluoroquinolones } \\
\hline Ciprofloxacin & 60.5 & 43.3 & 70 & & 40.1 \\
\hline Levofloxacin & 60.5 & 42.9 & 70.7 & & 40.8 \\
\hline Gatifloxacin & 56.3 & 39.9 & 5.2 & & 38 \\
\hline Garenoxacin & 51.3 & 37.4 & 62.1 & & 35.9 \\
\hline \multicolumn{6}{|l|}{ Others } \\
\hline Tetracycline & 3.4 & 0 & 1.7 & & 0 \\
\hline $\begin{array}{l}\text { Trimethoprim/ } \\
\text { sulfamethoxazole }\end{array}$ & 2.5 & 3.9 & 3.4 & & 7.7 \\
\hline Salmonella spp. & $\begin{array}{c}\text { Bacteremia } \\
(35)\end{array}$ & $\begin{array}{c}\text { Pneumonia } \\
\left({ }^{a}\right)\end{array}$ & $\begin{array}{c}\text { Bacteremia } \\
\left({ }^{(a)}\right.\end{array}$ & $\begin{array}{c}\text { Pneumonia } \\
\text { (a) }\end{array}$ & \\
\hline \multicolumn{6}{|l|}{ Cephalosporins } \\
\hline Cefazolin & 100 & a & a & a & \\
\hline Cefuroxime & 91.4 & a & a & a & \\
\hline Cefoxitin & 97.1 & a & a & a & \\
\hline Ceftriaxone & 100 & a & a & a & \\
\hline Ceftazidime & 100 & a & a & a & \\
\hline Cefepime & 100 & a & a & a & \\
\hline \multicolumn{6}{|l|}{ Other $\beta$-lactams } \\
\hline Ampicillin & 91.4 & a & a & a & \\
\hline Aztreonam & 100 & a & a & a & \\
\hline Ticarcillin/clavulanate & 91.4 & a & a & a & \\
\hline Piperacillin/tazobactam & 97.1 & a & a & a & \\
\hline Imipenem & 100 & a & a & a & \\
\hline Meropenem & 100 & a & a & a & \\
\hline \multicolumn{6}{|l|}{ Aminoglycosides } \\
\hline Amikacin & 100 & a & a & a & \\
\hline Gentamicin & 97.1 & a & a & a & \\
\hline Tobramycin & 97.1 & a & a & a & \\
\hline \multicolumn{6}{|l|}{ Fluoroquinolones } \\
\hline Ciprofloxacin & 100 & a & a & a & \\
\hline Levofloxacin & 100 & a & a & a & \\
\hline Gatifloxacin & 100 & a & a & a & \\
\hline Garenoxacin & 100 & a & a & a & \\
\hline \multicolumn{6}{|l|}{ Others } \\
\hline Tetracycline & 85.7 & a & a & a & \\
\hline $\begin{array}{l}\text { Trimethoprim/ } \\
\text { sulfamethoxazole }\end{array}$ & 100 & a & a & a & \\
\hline
\end{tabular}


Serratia spp.

Cephalosporins

Cefazolin

Cefuroxime

Cefoxitin

Ceftriaxone

Ceftazidime

Cefepime

Other $\beta$-lactams

Ampicillin

Aztreonam

Ticarcillin/clavulanate

Piperacillin/tazobactam

Imipenem

Meropenem

Aminoglycosides

Amikacin

Gentamicin

Tobramycin

Fluoroquinolones

Ciprofloxacin

Levofloxacin

Gatifloxacin

Garenoxacin

Others

Tetracycline

Trimethoprim/

sulfamethoxazole

\section{Bacteremia}

(32)

0

62.5

78.1

68.8

$$
0
$$

68.8

34.4

56.3

100

100

62.5

43.8

37.5

56.3

62.5

62.5

46.9

0

46.9

Bacteremia

(38)

Cefepime

Other $\beta$-lactams

Aztreonam

Ticarcillin/clavulanate

Piperacillin/tazobactam

Imipenem

Meropenem

Aminoglycosides

$$
\text { Amikacin }
$$

Gentamicin
0

0

65.8

39.5

Pneumonia

(18)

5.6

0

5.6

83.3

94.4

94.4

0

88.9

72.2

94.4

94.4

100

83.3

88.9

72.2

100

100

100

83.3

0

83.3

Pneumonia

(19)

52.6

26.3

5.3

52.6

29.7

0

2.6

0

47.4

15.8

0

5.3

10.5

13.2

10.5
Bacteremia Pneumonia

(22)

(a)

$0 \quad$ a

0

0

68.2

77.3

77.3

0

77.3

31.8

45.5

100

100

59.1

40.9

31.8

45.5

50

50

40.9

0

45.5

Bacteremia Pneumonia

(16)

(a)

93.8

62.5

0

81.3

43.8

0

6.3

18.8

18.8 


\begin{tabular}{ccccc}
\hline $\begin{array}{c}\text { Tobramycin } \\
\text { Fluoroquinolones }\end{array}$ & 10.5 & 5.3 & 18.8 & a \\
Ciprofloxacin & 68.4 & 42.1 & 62.5 & a \\
Levofloxacin & 100 & 100 & 100 & a \\
Gatifloxacin & 100 & 100 & 100 & a \\
$\quad$ Garenoxacin & 94.7 & 84.2 & 18.8 & a \\
Others & 15.8 & 5.3 & 100 & a \\
Tetracycline & 97.4 & 100 & & a \\
Trimethoprim/ & & & \\
sulfamethoxazole & 97.5 &
\end{tabular}

a. The results were not analyzed because the number of isolates was low $(<10)$;

b. Percentage of strains with MIC $\geq 2 \mu \mathrm{g} / \mathrm{mL}$, indicating possible ESBL production [6].

Table 5. Antimicrobial activity and spectrum of drugs tested against the most prevalent Gram-positive pathogens isolated in the year 2001 from patients with bacteremia and pneumonia

\begin{tabular}{lcccc}
\hline Pathogen/ & \multicolumn{2}{c}{ \% Susceptible strains (number of isolates tested) } \\
\cline { 2 - 5 } Antimicrobial agents & \multicolumn{2}{c}{ Latin America } & \multicolumn{2}{c}{ Brazil } \\
\hline S. aureus & $\begin{array}{c}\text { Bacteremia } \\
(\mathbf{3 8 9}\end{array}$ & $\begin{array}{c}\text { Pneumonia } \\
\text { (178) }\end{array}$ & $\begin{array}{c}\text { Bacteremia } \\
\text { (182) }\end{array}$ & $\begin{array}{c}\text { Pneumonia } \\
\text { (105) }\end{array}$ \\
Cephalosporins & & & & \\
Cefazolin & & & & \\
Ceftriaxone & $65^{\mathrm{b}}$ & $43.3^{\mathrm{b}}$ & $64.8^{\mathrm{b}}$ & $47.6^{\mathrm{b}}$ \\
Cefepime & $65^{\mathrm{b}}$ & $43.3^{\mathrm{b}}$ & $64.8^{\mathrm{b}}$ & $47.6^{\mathrm{b}}$ \\
Other $\beta$-lactams & $65^{\mathrm{b}}$ & $43.3^{\mathrm{b}}$ & $64.8^{\mathrm{b}}$ & $47.6^{\mathrm{b}}$ \\
Oxacillin & & & & \\
Penicillin & 65 & 43.3 & 64.8 & 47.6 \\
Amoxicillin/clavulanate & 10 & 2.8 & 12.6 & 3.8 \\
Piperacillin/tazobactam & $65^{\mathrm{b}}$ & $43.3^{\mathrm{b}}$ & $64.8^{\mathrm{b}}$ & $47.6^{\mathrm{b}}$ \\
Imipenem & $65^{\mathrm{b}}$ & $43.3^{\mathrm{b}}$ & $64.8^{\mathrm{b}}$ & $47.6^{\mathrm{b}}$ \\
MLS & $65^{\mathrm{b}}$ & $43.3^{\mathrm{b}}$ & $64.8^{\mathrm{b}}$ & $47.6^{\mathrm{b}}$ \\
Clindamycin & & & & \\
Erythromycin & 69.9 & 46.1 & 68.7 & 47.6 \\
Fluoroquinolones & 57.6 & 39.9 & 54.9 & 40 \\
Ciprofloxacin & & & & \\
Levofloxacin & 66.6 & 47.2 & 68.1 & 49.5 \\
Gatifloxacin & 71.2 & 51.1 & 73.1 & 54.3 \\
Garenoxacin & 91.3 & 87.1 & 88.5 & 84.8 \\
Others & 98.7 & 95.5 & 98.9 & 96.2 \\
Gentamicin & 63.2 & 44.9 & 63.7 & 47.6 \\
Rifampin & 78.7 & 65.7 & 74.7 & 62.9 \\
Chloramphenicol & & & & \\
\hline
\end{tabular}




\begin{tabular}{|c|c|c|c|c|}
\hline Tetracycline & 81.2 & 62.9 & 67 & 51.4 \\
\hline Doxycycline & 87.4 & 75.3 & 76.4 & 64.8 \\
\hline Trimethoprim/sulfamethoxale & 79.7 & 64 & 62.1 & 52.4 \\
\hline Vancomycin & 100 & 100 & 100 & 100 \\
\hline Teicoplanin & 99.7 & 100 & 99.5 & 100 \\
\hline Quinupristin/dalfopristin & 100 & 100 & 100 & 100 \\
\hline Linezolid & 100 & 100 & 100 & 100 \\
\hline CoNS & $\begin{array}{c}\text { Bacteremia } \\
\text { (219) }\end{array}$ & $\begin{array}{c}\text { Pneumonia } \\
\text { (11) }\end{array}$ & $\begin{array}{c}\text { Bacteremia } \\
(\mathbf{1 2 3})\end{array}$ & $\begin{array}{c}\text { Pneumonia } \\
\text { (11) }\end{array}$ \\
\hline \multicolumn{5}{|l|}{ Cephalosporins } \\
\hline Cefazolin & $19.6^{\mathrm{b}}$ & $54.5^{\mathrm{b}}$ & $15.4^{\mathrm{b}}$ & $54.5^{b}$ \\
\hline Ceftriaxone & $19.6^{b}$ & $54.5^{b}$ & $15.4^{\mathrm{b}}$ & $54.5^{b}$ \\
\hline Cefepime & $19.6^{\mathrm{b}}$ & $54.5^{\mathrm{b}}$ & $15.4^{\mathrm{b}}$ & $54.5^{b}$ \\
\hline \multicolumn{5}{|l|}{ Other $\beta$-lactams } \\
\hline Oxacillin & 19.6 & 54.5 & 15.4 & 54.5 \\
\hline Penicillin & 10.5 & 36.4 & 8.9 & 36.4 \\
\hline Amoxicillin/clavulanate & $19.6^{b}$ & $54.5^{b}$ & $15.4^{\mathrm{b}}$ & $54.5^{b}$ \\
\hline Piperacillin/tazobactam & $19.6^{b}$ & $54.5^{b}$ & $15.4^{\mathrm{b}}$ & $54.5^{b}$ \\
\hline Imipenem & $19.6^{b}$ & $54.5^{b}$ & $15.4^{\mathrm{b}}$ & $54.5^{b}$ \\
\hline \multicolumn{5}{|l|}{ MLS } \\
\hline Clindamycin & 55.7 & 63.6 & 49.6 & 63.6 \\
\hline Erythromycin & 30.1 & 27.3 & 30.9 & 27.3 \\
\hline \multicolumn{5}{|l|}{ Fluoroquinolones } \\
\hline Ciprofloxacin & 54.3 & 54.5 & 47.2 & 54.5 \\
\hline Gatifloxacin & 93.2 & 81.8 & 92.7 & 81.8 \\
\hline Levofloxacin & 65.3 & 54.5 & 61 & 54.5 \\
\hline Garenoxacin & 95.4 & 90.9 & 95.1 & 90.9 \\
\hline \multicolumn{5}{|l|}{ Others } \\
\hline Gentamicin & 45.9 & 54.5 & 40.2 & 54.5 \\
\hline Rifampin & 77.2 & 72.7 & 74 & 72.7 \\
\hline Chloramphenicol & 69.4 & 81.8 & 60.2 & 81.8 \\
\hline Tetracycline & 78.1 & 63.6 & 74 & 63.6 \\
\hline Doxycycline & 89 & 81.8 & 87.8 & 81.8 \\
\hline Trimethoprim/sulfamethoxale & 51.1 & 27.3 & 39.8 & 27.3 \\
\hline Vancomycin & 100 & 100 & 100 & 100 \\
\hline Teicoplanin & 93.2 & 81.8 & 92.7 & 81.8 \\
\hline Quinupristin/dalfopristin & 99.5 & 100 & 100 & 100 \\
\hline Linezolid & 100 & 100 & 100 & 100 \\
\hline Enterococcus spp. & $\begin{array}{c}\text { Bacteremia } \\
\qquad(64)\end{array}$ & $\begin{array}{c}\text { Pneumonia } \\
(\mathrm{d})\end{array}$ & $\begin{array}{c}\text { Bacteremia } \\
(28)\end{array}$ & $\begin{array}{c}\text { Pneumonia } \\
\left({ }^{d}\right)\end{array}$ \\
\hline \multicolumn{5}{|l|}{$\beta$-lactams } \\
\hline Ampicillin & 90.6 & d & 96.4 & d \\
\hline
\end{tabular}




\begin{tabular}{lllll}
\hline Penicillin & 76.6 & d & 64.3 & d \\
Amoxicillin/Clavulanate & $90.6^{\mathrm{c}}$ & d & $96.4^{\mathrm{c}}$ & $\mathrm{d}$ \\
Piperacillin/Tazobactam & $90.6^{\mathrm{c}}$ & $\mathrm{d}$ & $96.4^{\mathrm{c}}$ & $\mathrm{d}$ \\
MLS & & & & \\
Erythromycin & 7.8 & $\mathrm{~d}$ & 10.7 & $\mathrm{~d}$ \\
Fluoroquinolones & & & & \\
Ciprofloxacin & 50 & $\mathrm{~d}$ & 39.3 & $\mathrm{~d}$ \\
Levofloxacin & 62.5 & $\mathrm{~d}$ & 53.6 & $\mathrm{~d}$ \\
Gatifloxacin & 60.9 & $\mathrm{~d}$ & 53.6 & $\mathrm{~d}$ \\
Garenoxacin & 90.6 & $\mathrm{~d}$ & 89.3 & $\mathrm{~d}$ \\
Others & & & & $\mathrm{d}$ \\
Gentamicin (HL) & 60.9 & $\mathrm{~d}$ & 46.4 & $\mathrm{~d}$ \\
Streptomycin (HL) & 62.5 & $\mathrm{~d}$ & 75 & $\mathrm{~d}$ \\
Rifampin & 28.1 & $\mathrm{~d}$ & 21.4 & $\mathrm{~d}$ \\
Chloramphenicol & 62.5 & $\mathrm{~d}$ & 46.4 & $\mathrm{~d}$ \\
Tetracycline & 32.8 & $\mathrm{~d}$ & 35.7 & $\mathrm{~d}$ \\
Doxycycline & 42.2 & $\mathrm{~d}$ & 39.3 & $\mathrm{~d}$ \\
Vancomycin & 96.9 & $\mathrm{~d}$ & 100 & $\mathrm{~d}$ \\
Teicoplanin & 96.9 & $\mathrm{~d}$ & 100 & $\mathrm{~d}$ \\
Quinupristin/dalfopristin & 7.8 & $\mathrm{~d}$ & 7.1 & $\mathrm{~d}$ \\
Linezolid & 100 & $\mathrm{~d}$ & 100 & \\
\hline
\end{tabular}

a. Interpreted by NCCLS 2003 criteria, when available [6]; b. Susceptibility is predicted by the oxacillin result [6]; c. Susceptibility is predicted by the ampicillin result [6]; d. The results were not analyzed because the number of isolates was low $(<10)$. Abbreviations: CoNS - coagulase negative staphylococci; HL - High level aminoglycoside resistence screen; NA - Not applicable, there is no breakpoint established by the NCCLS [6].

Table 6. Antimicrobial activity and spectrum of drugs tested against the five most prevalent Gram-negative pathogens isolated from January 1997 to December 2001 throughout Latin America and in Brazil alone

\begin{tabular}{|c|c|c|c|c|}
\hline \multirow{2}{*}{$\begin{array}{l}\text { Pathogen (no total/Brazil)/ } \\
\text { Antimicrobial agent }\end{array}$} & \multicolumn{2}{|c|}{ Latin America } & \multicolumn{2}{|c|}{ Brazil } \\
\hline & $\mathrm{MIC}_{50}$ & $\mathrm{MIC}_{90}$ & $\%$ Susceptible & \% Susceptible \\
\hline \multicolumn{5}{|l|}{ Acinetobacter spp. (826/400) } \\
\hline \multicolumn{5}{|l|}{ Cephalosporins } \\
\hline Ceftazidime & $>16$ & $>16$ & 28.6 & 27.3 \\
\hline Cefepime & 16 & $>16$ & 35.4 & 33.8 \\
\hline \multicolumn{5}{|l|}{ Other $\beta$-lactams } \\
\hline Aztreonam & $>16$ & $>16$ & 7.6 & 6.5 \\
\hline Ticarcillin/clavulanate & $>128$ & $>128$ & 24.8 & 28.3 \\
\hline Piperacillin/tazobactam & $>64$ & $>64$ & 26.8 & 28.8 \\
\hline Imipenem & 1 & $>8$ & 86.9 & 90.8 \\
\hline Meropenem & 1 & $>8$ & 86.8 & 90.8 \\
\hline
\end{tabular}




\begin{tabular}{|c|c|c|c|c|}
\hline \multicolumn{5}{|l|}{ Aminoglycosides } \\
\hline Amikacin & $>32$ & $>32$ & 34 & 32.3 \\
\hline Gentamicin & $>8$ & $>16$ & 32.9 & 37.8 \\
\hline Tobramycin & 16 & $>16$ & 41.7 & 51.8 \\
\hline \multicolumn{5}{|l|}{ Fluoroquinolones } \\
\hline Ciprofloxacin & $>2$ & $>2$ & 30.5 & 35.3 \\
\hline Levofloxacin & $>4$ & $>4$ & 32.3 & 36.3 \\
\hline Gatifloxacin & 4 & $>4$ & 34.4 & 38.5 \\
\hline Garenoxacin & $>4$ & $>4$ & 34.9 & 35.8 \\
\hline \multicolumn{5}{|l|}{ Others } \\
\hline Tetracycline & $\leq 4$ & $>8$ & 51 & 56.1 \\
\hline $\begin{array}{l}\text { Trimethoprim/ } \\
\text { sulfamethoxazole }\end{array}$ & $>1$ & $>2$ & 32.4 & 31 \\
\hline \multicolumn{5}{|l|}{ Burkholderia cepacia (68/34) } \\
\hline \multicolumn{5}{|l|}{ Cephalosporins } \\
\hline Ceftazidime & 4 & 16 & 82.4 & 85.3 \\
\hline Cefepime & 8 & $>16$ & 51.5 & 47.1 \\
\hline \multicolumn{5}{|l|}{ Other $\beta$-lactams } \\
\hline Aztreonam & $>16$ & $>16$ & 19.1 & 20.6 \\
\hline Ticarcillin/clavulanate & $>128$ & $>128$ & 17.6 & 14.7 \\
\hline Piperacillin/tazobactam & 8 & 64 & 67.6 & 64.7 \\
\hline Imipenem & 4 & $>8$ & 52.9 & 52.9 \\
\hline Meropenem & 2 & $>8$ & 76.5 & 85.3 \\
\hline \multicolumn{5}{|l|}{ Aminoglycosides } \\
\hline Amikacin & $>32$ & $>32$ & 17.6 & 8.8 \\
\hline Gentamicin & 16 & $>16$ & 13.2 & 8.8 \\
\hline Tobramycin & $>16$ & $>16$ & 13.2 & 8.8 \\
\hline \multicolumn{5}{|l|}{ Fluoroquinolones } \\
\hline Ciprofloxacin & 1 & $>2$ & 60.3 & 70.6 \\
\hline Levofloxacin & 1 & 4 & 80.9 & 82.4 \\
\hline Gatifloxacin & 1 & $>4$ & 77.9 & 79.4 \\
\hline Garenoxacin & 2 & $>4$ & 66.7 & 76.9 \\
\hline \multicolumn{5}{|l|}{ Others } \\
\hline Tetracycline & $>8$ & $>8$ & 13.2 & 8.8 \\
\hline $\begin{array}{l}\text { Trimethoprim/ } \\
\text { sulfamethoxazole }\end{array}$ & $\leq 0.5$ & $>1$ & 79.4 & 97.1 \\
\hline \multicolumn{5}{|l|}{ Citrobacter spp. (136/43) } \\
\hline \multicolumn{5}{|l|}{ Cephalosporins } \\
\hline Cefazolin & $>16$ & $>16$ & 19.1 & 18.6 \\
\hline Cefuroxime & 8 & $>16$ & 56.6 & 55.8 \\
\hline Cefoxitin & $>32$ & $>32$ & 20.6 & 20.9 \\
\hline Ceftriaxone & 0.25 & $>32$ & 70.6 & 65.1 \\
\hline
\end{tabular}




\begin{tabular}{|c|c|c|c|c|}
\hline Ceftazidime & 1 & $>16$ & 67.6 & 62.8 \\
\hline Cefepime & 0.12 & 4 & 93.4 & 95.3 \\
\hline \multicolumn{5}{|l|}{ Other $\beta$-lactams } \\
\hline Ampicillin & $>16$ & $>16$ & 12.5 & 11.6 \\
\hline Aztreonam & 0.25 & $>16$ & 66.2 & 60.5 \\
\hline Ticarcillin/clavulanate & 4 & $>128$ & 59.6 & 55.8 \\
\hline Piperacillin/tazobactam & 4 & $>64$ & 68.4 & 67.4 \\
\hline Imipenem & 0.5 & 1 & 100 & 100 \\
\hline Meropenem & 0.06 & 0.12 & 100 & 100 \\
\hline \multicolumn{5}{|l|}{ Aminoglycosides } \\
\hline Amikacin & 2 & 32 & 81.6 & 74.4 \\
\hline Gentamicin & $\leq 1$ & 16 & 79.4 & 74.4 \\
\hline Tobramycin & 1 & $>16$ & 73.5 & 71.8 \\
\hline \multicolumn{5}{|l|}{ Fluoroquinolones } \\
\hline Ciprofloxacin & 0.25 & $>2$ & 81.6 & 76.7 \\
\hline Levofloxacin & $\leq 0.5$ & 4 & 84.6 & 81.4 \\
\hline Gatifloxacin & 0.12 & 4 & 83.1 & 76.7 \\
\hline Garenoxacin & 0.12 & $>4$ & 85.3 & 81 \\
\hline \multicolumn{5}{|l|}{ Others } \\
\hline Tetracycline & $\leq 4$ & $>8$ & 67.6 & 67.4 \\
\hline $\begin{array}{l}\text { Trimethoprim/ } \\
\text { sulfamethoxazole }\end{array}$ & $\leq 0.5$ & $>2$ & 68.4 & 62.8 \\
\hline \multicolumn{5}{|l|}{ Enterobacter spp. (897/ 441) } \\
\hline \multicolumn{5}{|l|}{ Cephalosporins } \\
\hline Cefazolin & $>16$ & $>16$ & 5.1 & 3.9 \\
\hline Cefuroxime & $>16$ & $>16$ & 35.9 & 40.4 \\
\hline Cefoxitin & $>32$ & $>32$ & 2.2 & 5.9 \\
\hline Ceftriaxone & 0.5 & $>32$ & 64.2 & 69.4 \\
\hline Ceftazidime & $\leq 1$ & $>16$ & 64 & 69.2 \\
\hline Cefepime & $\leq 0.12$ & 8 & 90.5 & 92.3 \\
\hline \multicolumn{5}{|l|}{ Other $\beta$-lactams } \\
\hline Ampicillin & $>16$ & $>16$ & 5.6 & 5.2 \\
\hline Aztreonam & 0.25 & $>16$ & 63.4 & 69.4 \\
\hline Ticarcillin/clavulanate & 16 & $>128$ & 52.1 & 55.8 \\
\hline Piperacillin/tazobactam & 4 & $>64$ & 64.9 & 68.3 \\
\hline Imipenem & 0.5 & 1 & 99.8 & 99.5 \\
\hline Meropenem & $\leq 0.06$ & 0.25 & 99.7 & 99.5 \\
\hline \multicolumn{5}{|l|}{ Aminoglycosides } \\
\hline Amikacin & 2 & $>32$ & 83.4 & 82.3 \\
\hline Gentamicin & $\leq 1$ & $>8$ & 74.1 & 74.6 \\
\hline Tobramycin & 1 & $>16$ & 63 & 66.8 \\
\hline \multicolumn{5}{|l|}{ Fluoroquinolones } \\
\hline Ciprofloxacin & 0.25 & $>2$ & 80.2 & 82.8 \\
\hline
\end{tabular}




\begin{tabular}{|c|c|c|c|c|}
\hline Levofloxacin & $\leq 0.5$ & $>4$ & 81.7 & 84.8 \\
\hline Gatifloxacin & 0.06 & $>4$ & 82.5 & 86.4 \\
\hline Garenoxacin & 0.12 & $>4$ & 75 & 85.6 \\
\hline \multicolumn{5}{|l|}{ Others } \\
\hline Tetracycline & $\leq 4$ & $>8$ & 66.6 & 67.8 \\
\hline $\begin{array}{l}\text { Trimethoprim/ } \\
\text { sulfamethoxazole }\end{array}$ & $\leq 0.5$ & $>2$ & 70.9 & 71.4 \\
\hline \multicolumn{5}{|l|}{ E. coli $(3164 / 835)$} \\
\hline \multicolumn{5}{|l|}{ Cephalosporins } \\
\hline Cefazolin & $\leq 2$ & $>16$ & 81.8 & 86 \\
\hline Cefuroxime & 4 & 16 & 88 & 89.1 \\
\hline Cefoxitin & 4 & 8 & 92.8 & 93.8 \\
\hline Ceftriaxone & $\leq 0.25$ & $\leq 0.25$ & $94.6(6.1)^{\mathrm{b}}$ & $94.3(6.7)^{b}$ \\
\hline Ceftazidime & 0.25 & $\leq 2$ & $95.8(6.5)^{b}$ & $95.3(7.4)^{b}$ \\
\hline Cefepime & $\leq 0.12$ & 0.25 & 96.9 & 96.9 \\
\hline \multicolumn{5}{|l|}{ Other $\beta$-lactams } \\
\hline Ampicillin & $>16$ & $>16$ & 39.7 & 42.9 \\
\hline Aztreonam & $\leq 0.12$ & 0.25 & $94.7(6.6)^{b}$ & $94.4(7)^{b}$ \\
\hline Ticarcillin/clavulanate & 8 & 128 & 65.2 & 72.7 \\
\hline Piperacillin/tazobactam & 2 & 16 & 91.3 & 94.3 \\
\hline Imipenem & 0.12 & 0.25 & 99.9 & 100 \\
\hline Meropenem & $\leq 0.06$ & $\leq 0.06$ & 99.9 & 100 \\
\hline \multicolumn{5}{|l|}{ Aminoglycosides } \\
\hline Amikacin & 2 & 8 & 97.3 & 97.6 \\
\hline Gentamicin & $\leq 1$ & 8 & 89 & 92.5 \\
\hline Tobramycin & 1 & 8 & 87 & 89.7 \\
\hline \multicolumn{5}{|l|}{ Fluoroquinolones } \\
\hline Ciprofloxacin & 0.25 & $>2$ & 82.9 & 89.7 \\
\hline Levofloxacin & $\leq 0.5$ & $>4$ & 82.9 & 90.3 \\
\hline Gatifloxacin & $\leq 0.03$ & $>4$ & 83.5 & 90.5 \\
\hline Garenoxacin & 0.03 & $>4$ & 84.4 & 91.2 \\
\hline \multicolumn{5}{|l|}{ Others } \\
\hline Tetracycline & $\leq 4$ & $>8$ & 51.4 & 58.7 \\
\hline $\begin{array}{l}\text { Trimethoprim/ } \\
\text { sulfamethoxazole }\end{array}$ & $\leq 0.5$ & $>2$ & 52.5 & 52.3 \\
\hline \multicolumn{5}{|c|}{ Haemophilus influenzae (1176/406) } \\
\hline \multicolumn{5}{|c|}{ Cephalosporins } \\
\hline Cefuroxime & 1 & 2 & 98.6 & 97.8 \\
\hline Ceftriaxone & 0.01 & 0.25 & 99.8 & 100 \\
\hline Ceftazidime & 0.25 & 0.25 & 99.8 & 100 \\
\hline Cefepime & 0.06 & 0.12 & 99.8 & 100 \\
\hline Other $\beta$-lactams & & & & \\
\hline
\end{tabular}




\begin{tabular}{|c|c|c|c|c|}
\hline Ampicillin & $\leq 0.5$ & $>4$ & $86.4^{\mathrm{c}}$ & $87.4^{\mathrm{c}}$ \\
\hline Aztreonam & 0.12 & 0.12 & 100 & 100 \\
\hline Piperacillin/tazobactam & $\leq 0.5$ & $\leq 0.5$ & 99.3 & 100 \\
\hline Imipenem & 0.25 & 0.5 & 100 & 100 \\
\hline Meropenem & 0.06 & 0.06 & 100 & 100 \\
\hline \multicolumn{5}{|l|}{ Macrolides } \\
\hline Azithromycin & 1 & 2 & 99.7 & 99.5 \\
\hline Clarithromycin & 8 & 8 & 91.1 & 90.8 \\
\hline \multicolumn{5}{|l|}{ Fluoroquinolones } \\
\hline Ciprofloxacin & 0.01 & 0.03 & 100 & 100 \\
\hline Gatifloxacin & 0.03 & 0.03 & 100 & 100 \\
\hline Levofloxacin & 0.03 & $\leq 0.5$ & 100 & 100 \\
\hline Garenoxacin & 0.03 & 0.03 & 100 & 100 \\
\hline \multicolumn{5}{|l|}{ Others } \\
\hline Tetracycline & $\leq 2$ & $\leq 2$ & 96.9 & 92.4 \\
\hline Choranphenicol & $\leq 2$ & $\leq 2$ & 97.5 & 96.4 \\
\hline $\begin{array}{l}\text { Trimethoprim/ } \\
\text { sulfamethoxazole }\end{array}$ & $\leq 0.5$ & $>4$ & 61 & 51.9 \\
\hline \multicolumn{5}{|l|}{ K. pneumoniae (1517/575) } \\
\hline \multicolumn{5}{|l|}{ Cephalosporins } \\
\hline Cefazolin & 16 & $>16$ & 49.6 & 46.3 \\
\hline Cefuroxime & 8 & $>16$ & 54 & 51.3 \\
\hline Cefoxitin & 4 & 16 & 83.6 & 84.5 \\
\hline Ceftriaxone & $\leq 0.25$ & $>32$ & $61.8(42.5)^{\mathrm{b}}$ & $57.9(47.8)^{\mathrm{b}}$ \\
\hline Ceftazidime & $\leq 2$ & $>16$ & $66.8(42.7)^{\mathrm{b}}$ & $67.5(48)^{\mathrm{b}}$ \\
\hline Cefepime & $\leq 0.12$ & $>16$ & 76.3 & 67.1 \\
\hline \multicolumn{5}{|l|}{ Other $\beta$-lactams } \\
\hline Ampicillin & $>16$ & $>16$ & 0 & 0 \\
\hline Aztreonam & 0.25 & $>16$ & $62.2(41.7)^{\mathrm{b}}$ & $57.7(46.3)^{b}$ \\
\hline Ticarcillin/clavulanate & 16 & $>128$ & 50.4 & 49 \\
\hline Piperacillin/tazobactam & 4 & $>64$ & 66.8 & 68.5 \\
\hline Imipenem & 0.25 & 0.5 & 99.8 & 100 \\
\hline Meropenem & $\leq 0.06$ & 0.12 & 99.4 & 99.5 \\
\hline \multicolumn{5}{|l|}{ Aminoglycosides } \\
\hline Amikacin & 2 & 32 & 80.7 & 81.6 \\
\hline Gentamicin & $\leq 1$ & $>16$ & 65.1 & 63.1 \\
\hline Tobramycin & 1 & $>16$ & 53.9 & 51.3 \\
\hline \multicolumn{5}{|l|}{ Fluoroquinolones } \\
\hline Ciprofloxacin & 0.25 & $>2$ & 86.5 & 88.9 \\
\hline Levofloxacin & $\leq 0.5$ & 4 & 88.7 & 89.6 \\
\hline
\end{tabular}




\begin{tabular}{|c|c|c|c|c|}
\hline Gatifloxacin & 0.06 & 2 & 89.8 & 90.3 \\
\hline Garenoxacin & 0.12 & $>4$ & 87.1 & 85.6 \\
\hline \multicolumn{5}{|l|}{ Others } \\
\hline Tetracycline & $\leq 4$ & $>8$ & 65.6 & 69.7 \\
\hline Choranphenicol & $\leq 2$ & $\leq 2$ & 99.7 & 99.3 \\
\hline $\begin{array}{l}\text { Trimethoprim/ } \\
\text { sulfamethoxazole }\end{array}$ & $\leq 0.5$ & 2 & 68.2 & 62.1 \\
\hline \multicolumn{5}{|c|}{ Moraxella catarrhalis $(379 / 141)$} \\
\hline \multicolumn{5}{|l|}{ Beta-lactams } \\
\hline Ampicillin & $\leq 2$ & 4 & $4.5^{\mathrm{c}}$ & $5^{c}$ \\
\hline Cefuroxime & 1 & 2 & 99.5 & 100 \\
\hline Ceftriaxone & 0.25 & 0.5 & 100 & 100 \\
\hline Ceftazidime & 0.25 & 0.25 & 99.6 & 100 \\
\hline Cefepime & 0.5 & 2 & 98.2 & 97.2 \\
\hline \multicolumn{5}{|l|}{ Macrolides } \\
\hline Azithromycin & $\leq 0.12$ & $\leq 0.12$ & 100 & 100 \\
\hline Clarithromycin & $\leq 0.25$ & $\leq 0.25$ & 100 & 100 \\
\hline \multicolumn{5}{|l|}{ Fluoroquinolones } \\
\hline Ciprofloxacin & 0.03 & 0.06 & 100 & 100 \\
\hline Levofloxacin & 0.03 & $\leq 0.5$ & 100 & 100 \\
\hline Gatifloxacin & 0.03 & 0.06 & 100 & 100 \\
\hline Garenoxacin & 0.03 & 0.03 & 100 & 100 \\
\hline \multicolumn{5}{|l|}{ Others } \\
\hline Tetracycline & $\leq 2$ & $\leq 2$ & 92.9 & 100 \\
\hline $\begin{array}{l}\text { Trimethoprim/ } \\
\text { sulfamethoxazole }\end{array}$ & $\leq 0.5$ & 1 & 89.1 & 86.5 \\
\hline \multicolumn{5}{|c|}{ Morganella morgannii (80/ 29) } \\
\hline \multicolumn{5}{|l|}{ Cephalosporins } \\
\hline Cefazolin & $>16$ & $>16$ & 3.8 & 6.9 \\
\hline Cefuroxime & $>16$ & $>16$ & 10 & 10.3 \\
\hline Cefoxitin & 16 & $>32$ & 43.8 & 37.9 \\
\hline Ceftriaxone & 0.25 & 4 & 92.5 & 82.8 \\
\hline Ceftazidime & 0.25 & 8 & 90 & 93.1 \\
\hline Cefepime & 0.12 & 2 & 95 & 89.7 \\
\hline \multicolumn{5}{|l|}{ Other $\beta$-lactams } \\
\hline Ampicillin & $>16$ & $>16$ & 0 & 0 \\
\hline Aztreonam & 0.12 & 16 & 87.5 & 82.8 \\
\hline Ticarcillin/clavulanate & 4 & 64 & 82.5 & 86.2 \\
\hline Piperacillin/tazobactam & $\leq 0.5$ & 8 & 92.5 & 96.6 \\
\hline Imipenem & 2 & 4 & 97.5 & 93.1 \\
\hline Meropenem & 0.06 & 0.25 & 100 & 100 \\
\hline Aminoglycosides & & & & \\
\hline
\end{tabular}


Fluoroquinolones

Ciprofloxacin

Levofloxacin

Gatifloxacin

Garenoxacin

Others

Tetracycline

Trimethoprim/

sulfamethoxazole
0.25

$\leq 0.5$

0.12

0.5

$\leq 4$

$\leq 0.5$

\section{$>2$}

$>4$

$>4$

$>4$

$>8$

$>2$

67.5
69

72.4

69

40

37.9

58.6

\section{P. aeruginosa (1984/ 913)}

Cephalosporins

Ceftazidime

Cefepime

4

8

$>16$

62.4

56

Other $\beta$-lactams

Aztreonam

16

$>16$

62.7

53.9

Ticarcillin/clavulanate

64

$>16$

44.5

38.2

Piperacillin/tazobactam

$>128$

55.6

47.8

Imipenem

$>64$

72.1

64.6

71.9

62.5

Meropenem

$>8$

74.8

65.8

Aminoglycosides

Amikacin

Gentamicin

Tobramycin

Fluoroquinolones

Ciprofloxacin

Levofloxacin

Gatifloxacin

Garenoxacin

Others

Tetracycline

Trimethoprim/

sulfamethoxazole

$>8$

70

58.4

58.2

49.7

58.2

47.9

$>16$

58.1

52.8

56.8

52.4

54.4

50.2

46.3

42

$>4$

1.5

0.9

$>2$

$>2$

3.4

3.8

\section{Proteus spp (328/ 66)}

Cephalosporins

Cefazolin

Cefuroxime

Cefoxitin

Ceftriaxone

Ceftazidime

Cefepime

Other $\beta$-lactams

Ampicillin

\section{4}

2

4

0.25

0.12

0.12

4
$>16$

$>16$

8

$>32$

2

$>16$
62.2

69.8

93.9

81.4

96.3

83.2

$>16$

52.4

74.2

78.8

95.5

92.4

98.5

92.4

56.1 


\begin{tabular}{lcccc}
\hline Aztreonam & 0.12 & 4 & 93.6 & 95.5 \\
Ticarcillin/clavulanate & $\leq 1$ & 16 & 94.5 & 97 \\
Piperacillin/tazobactam & $\leq 0.5$ & 2 & 94.2 & 98.5 \\
Imipenem & 1 & 4 & 98.8 & 98.5 \\
Meropenem & 0.06 & 0.25 & 100 & 100 \\
Aminoglycosides & & & & \\
Amikacin & 4 & 16 & 93.6 & 97 \\
Gentamicin & 1 & $>16$ & 74.4 & 87.9 \\
Tobramycin & 2 & 16 & 75.6 & 87.7 \\
Fluoroquinolones & & & & 92.4 \\
Ciprofloxacin & 0.25 & $>2$ & 76.2 & 95.5 \\
Levofloxacin & $\leq 0.5$ & $>4$ & 79.6 & 87.8 \\
Gatifloxacin & 0.12 & $>4$ & 76.8 & \\
Garenoxacin & 0.5 & & 75.1 & \\
Others & & $>8$ & 5.5 & 71.2 \\
Tetracycline & $>8$ & & 61.6 & \\
Trimethoprim/ & & & & \\
sulfamethoxazole & $\leq 0.5$ & & &
\end{tabular}

\section{Salmonella spp (308/97)}

Cephalosporins

Cefazolin

Cefuroxime

Cefoxitin

Ceftriaxone

Ceftazidime

Cefepime

Other $\beta$-lactams

Ampicillin

Aztreonam

Ticarcillin/clavulanate

Piperacillin/tazobactam

Imipenem

Meropenem

Aminoglycosides

Amikacin

Gentamicin

Tobramycin

Fluoroquinolones

Ciprofloxacin

Levofloxacin

Gatifloxacin

Garenoxacin

Others $\leq 2$

4

2

0.25

$\leq 1$

0.12

$\leq 2$

0.12

2

2

0.25

0.06

1

$\leq 1$

1

0.01

0.06

0.03

0.06
96.8

96.8

97.7

100

100

100

0.12

16

0.12

8

4

0.25

0.06

89.3

99

92.5

99.4

100

100

99.7

96.4

95.7

99

96.9

97.9

100

100

100

96.9

100

100

100

100

100

100

95.8

94.3

100

100

100

100 


\begin{tabular}{lcccc}
\hline Tetracycline & $\leq 4$ & 8 & 86.4 & 94.8 \\
$\begin{array}{l}\text { Trimethoprim/ } \\
\text { sulfamethoxazole }\end{array}$ & $\leq 0.5$ & $\leq 0.5$ & 93.8 & 97.9
\end{tabular}

Serratia spp. (320/ 169)

Cephalosporins

Cefazolin

Cefuroxime

Cefoxitin

Ceftriaxone

Ceftazidime

Cefepime

$$
>16
$$

$>16$

32

0.5

0.5

0.25

Other $\beta$-lactams

Ampicillin

Ticarcillin/clavulanate

Piperacillin/tazobactam

Imipenem

Meropenem

Aminoglycosides

Amikacin

Gentamicin

Tobramycin

Fluoroquinolones

Ciprofloxacin

Levofloxacin

Gatifloxacin

Garenoxacin

\section{Others}

Tetracycline

Trimethoprim/

sulfamethoxazole
Aztreonam

$\begin{array}{cc}>16 & >16 \\ 0.25 & >16 \\ 16 & >128 \\ 2 & >64 \\ 1 & 2 \\ 0.06 & 0.25 \\ & \\ 4 & >32 \\ 1 & >16 \\ 4 & >16\end{array}$

0.25

$\leq 0.5$

0.25

2

$>8$

$\leq 0.5$

$>16$

$>16$

64

0.25

$>32$

$>16$

$>16$

$>2$

4

$>4$

$>4$

$>8$

$>2$
$>16$

$>16$

$>32$

$>32$

$>16$

16

128

73.4

66.6

54.4

74.1

82.2

80

62.5

7.5

Shigella spp (209/ 87)

Cephalosporins

Cefazolin

Cefuroxime

Cefoxitin

Ceftriaxone

Ceftazidime

Cefepime

Other $\beta$-lactams

Ampicillin

Aztreonam $\leq 2$

2

2

0.25

$\leq 2$

0.12

$>16$

0.12
4

4

4

0.25

$\leq 2$

0.25

$>16$

0.12
98.6

99

99

99.5

100

100
58.6

1.2

0.6

6.5

71.6

81.1

93.5

3

84

47.9

64.5

100

100

66.9

56.2

45

59.8

69.8

67.5

51.7

4.7

98.9

98.9

98.9

100

100

100 


\begin{tabular}{|c|c|c|c|c|}
\hline Ticarcillin/clavulanate & 16 & 32 & 82.8 & 86.2 \\
\hline Piperacillin/tazobactam & 2 & 2 & 99 & 100 \\
\hline Imipenem & 0.12 & 0.25 & 100 & 100 \\
\hline Meropenem & 0.06 & 0.06 & 100 & 100 \\
\hline \multicolumn{5}{|l|}{ Aminoglycosides } \\
\hline Amikacin & 8 & 8 & 100 & 100 \\
\hline Gentamicin & $\leq 1$ & 2 & 100 & 100 \\
\hline Tobramycin & 1 & 2 & 100 & 100 \\
\hline \multicolumn{5}{|l|}{ Fluoroquinolones } \\
\hline Ciprofloxacin & 0.01 & 0.01 & 100 & 100 \\
\hline Levofloxacin & 0.03 & 0.03 & 100 & 100 \\
\hline Gatifloxacin & 0.03 & 0.03 & 100 & 100 \\
\hline Garenoxacin & 0.03 & 0.03 & 100 & 100 \\
\hline \multicolumn{5}{|l|}{ Others } \\
\hline Tetracycline & $\leq 4$ & $>8$ & 53.1 & 71.3 \\
\hline $\begin{array}{l}\text { Trimethoprim/ } \\
\text { sulfamethoxazole }\end{array}$ & $>2$ & $>2$ & 25.8 & 14.9 \\
\hline \multicolumn{5}{|c|}{ Stenotrophomonas maltophilia (166/ 62) } \\
\hline \multicolumn{5}{|l|}{ Cephalosporins } \\
\hline Ceftazidime & 8 & $>16$ & 57.8 & 66.1 \\
\hline Cefepime & 16 & $>16$ & 31.3 & 35.5 \\
\hline \multicolumn{5}{|l|}{ Other $\beta$-lactams } \\
\hline Aztreonam & $>16$ & $>16$ & 5.4 & 4.8 \\
\hline Ticarcillin/clavulanate & 16 & 128 & 56 & 69.4 \\
\hline Piperacillin/tazobactam & 64 & $>64$ & 33.3 & 40.3 \\
\hline Imipenem & $>8$ & $>8$ & 1.2 & 0 \\
\hline Meropenem & $>8$ & $>8$ & 4.8 & 6.5 \\
\hline \multicolumn{5}{|l|}{ Aminoglycosides } \\
\hline Amikacin & $>32$ & $>32$ & 16.3 & 12.9 \\
\hline Gentamicin & $>8$ & $>16$ & 15.1 & 11.3 \\
\hline Tobramycin & $>16$ & $>16$ & 13.1 & 8.1 \\
\hline \multicolumn{5}{|l|}{ Fluoroquinolones } \\
\hline Ciprofloxacin & 2 & $>2$ & 43.4 & 33.9 \\
\hline Levofloxacin & 0.5 & 4 & 88 & 90.3 \\
\hline Gatifloxacin & 0.5 & 2 & 91 & 93.5 \\
\hline Garenoxacin & 1 & 4 & 82.4 & 78.4 \\
\hline \multicolumn{5}{|l|}{ Others } \\
\hline Tetracycline & $>8$ & $>8$ & 14.5 & 12.9 \\
\hline $\begin{array}{l}\text { Trimethoprim/ } \\
\text { sulfamethoxazole }\end{array}$ & $\leq 0.5$ & $\leq 0.5$ & 97.1 & 100 \\
\hline
\end{tabular}

a. The results were not analyzed because the number of isolates was low $(<10)$; b. Percentage of strains with $\mathrm{MIC} \geq 2 \mu \mathrm{g} / \mathrm{mL}$, indicating possible ESBL production [6]; c. Percentage of non-beta-lactamase producing strains. This value also represents the susceptbility rate to amoxicillin [6]. 
Table 7. Antimicrobial activity and spectrum of drugs tested against the most prevalent Gram-positive cocci isolated from January 1997 to December 2001 throughout Latin America and in Brazil alone

\begin{tabular}{|c|c|c|c|c|}
\hline \multirow{2}{*}{$\begin{array}{l}\text { Pathogen (no total/Brazil)/ } \\
\text { Antimicrobial agent }\end{array}$} & \multicolumn{2}{|c|}{ Latin America } & \multicolumn{2}{|c|}{ Brazil } \\
\hline & $\mathrm{MIC}_{50}$ & $\mathrm{MIC}_{90}$ & $\%$ Susceptible & $\%$ Susceptible \\
\hline \multicolumn{5}{|l|}{ S. aureus (3396/ 1516) } \\
\hline \multicolumn{5}{|l|}{ Cephalosporins } \\
\hline Cefazolin & $\leq 2$ & $>16$ & $63.2^{\mathrm{b}}$ & $62.7^{\mathrm{b}}$ \\
\hline Ceftriaxone & 4 & $>32$ & $63.2^{\mathrm{b}}$ & $62.7^{\mathrm{b}}$ \\
\hline Cefepime & 8 & $>16$ & $63.2^{\mathrm{b}}$ & $62.7^{b}$ \\
\hline \multicolumn{5}{|l|}{ Other $\beta$-lactams } \\
\hline Oxacillin & 0.5 & $>8$ & 63.2 & 62.7 \\
\hline Penicillin & 16 & $>32$ & 7.2 & 9 \\
\hline Amoxicillin/clavulanate & 2 & $>16$ & $63.2^{b}$ & $62.7^{b}$ \\
\hline Piperacillin/tazobactam & 2 & $>64$ & $63.2^{\mathrm{b}}$ & $62.7^{\mathrm{b}}$ \\
\hline Imipenem & $\leq 0.06$ & $>8$ & $63.2^{\mathrm{b}}$ & $62.7^{b}$ \\
\hline \multicolumn{5}{|l|}{ MLS } \\
\hline Clindamycin & 0.25 & $>8$ & 66 & 64.1 \\
\hline Erythromycin & 0.5 & $>8$ & 50.5 & 47.9 \\
\hline \multicolumn{5}{|l|}{ Fluoroquinolones } \\
\hline Ciprofloxacin & 0.5 & $>2$ & 64.5 & 63.4 \\
\hline Levofloxacin & 0.25 & 4 & 66.3 & 64.8 \\
\hline Gatifloxacin & 0.12 & 4 & 89.6 & 87.8 \\
\hline Garenoxacin & 0.03 & 2 & 97.6 & 96.9 \\
\hline \multicolumn{5}{|l|}{ Others } \\
\hline Gentamicin & $\leq 1$ & $>16$ & 62.7 & 61.9 \\
\hline Rifampin & $\leq 0.25$ & 2 & 76.6 & 67.8 \\
\hline Chloramphenicol & 8 & $>16$ & 64.4 & 63.2 \\
\hline Tetracycline & $\leq 4$ & $>8$ & 71.1 & 60.9 \\
\hline Doxycycline & $\leq 0.5$ & $>4$ & 83.7 & 75.2 \\
\hline Trimethoprim/sulfamethoxale & $\mathrm{e} \leq 0.5$ & $>1$ & 75.8 & 63.5 \\
\hline Vancomycin & 1 & 1 & 100 & 100 \\
\hline Teicoplanin & 1 & 2 & 99.6 & 99.6 \\
\hline Quinupristin/dalfopristin & 0.25 & 0.5 & 99.7 & 99.9 \\
\hline Linezolid & 2 & 4 & 100 & 100 \\
\hline \multicolumn{5}{|l|}{ CoNS (1397/ 514) } \\
\hline \multicolumn{5}{|l|}{ Cephalosporins } \\
\hline Cefazolin & $\leq 2$ & $>16$ & $22.8^{b}$ & $19.5^{\mathrm{b}}$ \\
\hline Ceftriaxone & 16 & $>32$ & $22.8^{b}$ & $19.5^{\mathrm{b}}$ \\
\hline Cefepime & 16 & $>16$ & $22.8^{b}$ & $19.5^{\mathrm{b}}$ \\
\hline \multicolumn{5}{|l|}{ Other $\beta$-lactams } \\
\hline Oxacillin & 8 & $>8$ & 22.8 & 19.5 \\
\hline
\end{tabular}




\begin{tabular}{|c|c|c|c|c|}
\hline Penicillin & 8 & $>32$ & 9.3 & 8.2 \\
\hline Amoxicillin/clavulanate & $\leq 2$ & $>16$ & $22.8^{b}$ & $19.5^{b}$ \\
\hline Piperacillin/tazobactam & 2 & $>64$ & $22.8^{b}$ & $19.5^{b}$ \\
\hline Imipenem & 0.5 & $>8$ & $22.8^{b}$ & $19.5^{b}$ \\
\hline \multicolumn{5}{|l|}{ MLS } \\
\hline Clindamycin & 0.25 & $>8$ & 55.3 & 46.3 \\
\hline Erythromycin & $>8$ & $>8$ & 35.3 & 34.6 \\
\hline \multicolumn{5}{|l|}{ Fluoroquinolones } \\
\hline Ciprofloxacin & 0.5 & $>2$ & 56.9 & 47.9 \\
\hline Levofloxacin & 0.25 & $>4$ & 65.7 & 58.1 \\
\hline Gatifloxacin & 0.12 & 2 & 93.8 & 92.8 \\
\hline Garenoxacin & 0.03 & 2 & 91.5 & 92.4 \\
\hline \multicolumn{5}{|l|}{ Others } \\
\hline Gentamicin & 8 & $>16$ & 45.1 & 40.5 \\
\hline Rifampin & 0.25 & $>2$ & 72 & 68.1 \\
\hline Chloramphenicol & 8 & $>16$ & 62.7 & 55.6 \\
\hline Tetracycline & $\leq 4$ & $>8$ & 76.5 & 74.9 \\
\hline Doxycycline & 1 & $>4$ & 87.1 & 85.8 \\
\hline Trimetoprim/sulfametoxale & 1 & $>2$ & 50.6 & 38.4 \\
\hline Vancomycin & 2 & 2 & 100 & 100 \\
\hline Teicoplanin & 2 & 8 & 90.3 & 90.3 \\
\hline Quinupristin/dalfopristin & 0.25 & 0.5 & 98.7 & 98.2 \\
\hline Linezolid & 1 & 2 & 100 & 100 \\
\hline \multicolumn{5}{|l|}{ Enterococcus spp. (583/ 243) } \\
\hline \multicolumn{5}{|l|}{$\beta$-lactams } \\
\hline Ampicillin & 1 & 4 & 94.7 & 97.5 \\
\hline Penicillin & 2 & 16 & 90.2 & 81.5 \\
\hline Amoxicillin/Clavulanate & $\leq 2$ & 4 & $94.7^{\mathrm{c}}$ & $97.5^{\mathrm{c}}$ \\
\hline Piperacillin/Tazobactam & 4 & $>64$ & $94.7^{\mathrm{c}}$ & $97.5^{\mathrm{c}}$ \\
\hline \multicolumn{5}{|l|}{ MLS } \\
\hline Erythromycin & $>8$ & $>8$ & 9.4 & 9.1 \\
\hline \multicolumn{5}{|l|}{ Fluoroquinolones } \\
\hline Ciprofloxacin & 1 & $>2$ & 52.7 & 46.9 \\
\hline Levofloxacin & 1 & $>4$ & 65.7 & 56.3 \\
\hline Gatifloxacin & 0.5 & $>4$ & 73.9 & 66.7 \\
\hline Garenoxacin & 0.25 & 4 & 93.6 & 94.9 \\
\hline \multicolumn{5}{|l|}{ Others } \\
\hline Gentamicin (HL) & $\leq 500$ & $>1,000$ & 76.2 & 65 \\
\hline Streptomycin (HL) & $\leq 1,000$ & $\leq 1,000$ & 90.1 & 94.7 \\
\hline Rifampin & $>2$ & $>2$ & 23.3 & 18.1 \\
\hline Chloramphenicol & 8 & $>16$ & 67.2 & 55.6 \\
\hline Tetracycline & $>8$ & $>8$ & 33.4 & 32.1 \\
\hline Doxycycline & $>4$ & $>4$ & 44.4 & 49.4 \\
\hline
\end{tabular}




\begin{tabular}{lccll}
\hline Vancomycin & 1 & 2 & 97.5 & 97.5 \\
Teicoplanin & 0.25 & 0.5 & 98.1 & 97.5 \\
Linezolid & 2 & 2 & 96.4 & 97 \\
\hline
\end{tabular}

a. Interpreted by NCCLS 2003 criteria, when available [6]; b. Susceptibility is predicted by the oxacillin result [6]; c. Susceptibility is predicted by the ampicillin result [6]; Abbreviations: HL - High level aminoglycoside resistance screen.

Table 8. Antimicrobial activity and spectrum of drugs tested against the most prevalent Gram-negative pathogens isolated from January 1997 to December 2001 throughout Latin America and in Brazil alone, from patients with bacteremia and pneumonia

\begin{tabular}{|c|c|c|c|c|}
\hline \multirow{3}{*}{$\begin{array}{l}\text { Pathogen/ } \\
\text { Antimicrobial agent } \\
\text { Acinetobacter spp. }\end{array}$} & \multicolumn{4}{|c|}{$\%$ Susceptible strains ${ }^{\text {a }}$ (number of isolates tested) } \\
\hline & \multicolumn{2}{|c|}{ Latin America } & \multicolumn{2}{|c|}{ Brazil } \\
\hline & $\begin{array}{c}\text { Bacteremia } \\
\text { (380) }\end{array}$ & $\begin{array}{c}\text { Pneumonia } \\
\text { (299) }\end{array}$ & $\begin{array}{c}\text { Bacteremia } \\
(212)\end{array}$ & $\begin{array}{c}\text { Pneumonia } \\
\text { (139) }\end{array}$ \\
\hline \multicolumn{5}{|l|}{ Cephalosporins } \\
\hline Ceftazidime & 40 & 17.7 & 40.6 & 12.2 \\
\hline Cefepime & 46.1 & 25.8 & 45.8 & 19.4 \\
\hline \multicolumn{5}{|l|}{ Other $\beta$-lactams } \\
\hline Aztreonam & 11.8 & 4.3 & 10.8 & 2.2 \\
\hline Ticarcillin/clavulanate & 35.8 & 13.4 & 40.1 & 12.9 \\
\hline Piperacillin/tazobactam & 37.6 & 16.4 & 39.6 & 15.1 \\
\hline Imipenem & 88.9 & 85.3 & 91.5 & 89.2 \\
\hline Meropenem & 89.5 & 84.3 & 92.5 & 88.5 \\
\hline \multicolumn{5}{|l|}{ Aminoglycosides } \\
\hline Amikacin & 43.2 & 23.1 & 43.9 & 17.3 \\
\hline Gentamicin & 44.2 & 23.4 & 49.5 & 23.7 \\
\hline Tobramycin & 52.4 & 32.7 & 64.7 & 36.2 \\
\hline \multicolumn{5}{|l|}{ Fluoroquinolones } \\
\hline Ciprofloxacin & 42.9 & 18.7 & 46.7 & 20.1 \\
\hline Levofloxacin & 45 & 21.1 & 48.6 & 20.1 \\
\hline Gatifloxacin & 45.5 & 24.1 & 48.6 & 25.2 \\
\hline Garenoxacin & 48.9 & 24.5 & 48.7 & 19.7 \\
\hline \multicolumn{5}{|l|}{ Others } \\
\hline Tetracycline & 59.9 & 46.2 & 65.9 & 46 \\
\hline $\begin{array}{l}\text { Trimethoprim/ } \\
\text { sulfamethoxazole }\end{array}$ & 42.4 & 23.4 & 40.6 & 19.4 \\
\hline Burkholderia cepacia & $\begin{array}{c}\text { Bacteremia } \\
\qquad(43)\end{array}$ & $\begin{array}{c}\text { Pneumonia } \\
\text { (19) }\end{array}$ & $\begin{array}{c}\text { Bacteremia } \\
\qquad(21)\end{array}$ & $\begin{array}{c}\text { Pneumonia } \\
\text { (b) }\end{array}$ \\
\hline \multicolumn{5}{|l|}{ Cephalosporins } \\
\hline Ceftazidime & 79.1 & 84.2 & 85.7 & $\mathrm{~b}$ \\
\hline Cefepime & 48.8 & 57.9 & 33.3 & $\mathrm{~b}$ \\
\hline
\end{tabular}




\begin{tabular}{|c|c|c|c|c|}
\hline \multicolumn{5}{|l|}{ Other $\beta$-lactams } \\
\hline Aztreonam & 16.3 & 15.8 & 19 & $\mathrm{~b}$ \\
\hline Ticarcillin/clavulanate & 14 & 26.3 & 9.5 & $\mathrm{~b}$ \\
\hline Piperacillin/tazobactam & 62.8 & 84.2 & 52.4 & $\mathrm{~b}$ \\
\hline Imipenem & 48.8 & 57.9 & 38.1 & $\mathrm{~b}$ \\
\hline Meropenem & 79.1 & 68.4 & 85.7 & $\mathrm{~b}$ \\
\hline \multicolumn{5}{|l|}{ Aminoglycosides } \\
\hline Amikacin & 16.3 & 21.1 & 4.8 & $\mathrm{~b}$ \\
\hline Gentamicin & 7 & 26.3 & 4.8 & $\mathrm{~b}$ \\
\hline Tobramycin & 9.3 & 21.1 & 4.8 & $\mathrm{~b}$ \\
\hline \multicolumn{5}{|l|}{ Fluoroquinolones } \\
\hline Ciprofloxacin & 65.1 & 47.4 & 71.4 & $\mathrm{~b}$ \\
\hline Gatifloxacin & 76.7 & 78.9 & 76.2 & $\mathrm{~b}$ \\
\hline Levofloxacin & 81.4 & 84.2 & 81 & $\mathrm{~b}$ \\
\hline Garenoxacin & 62.5 & 75 & 66.7 & $\mathrm{~b}$ \\
\hline \multicolumn{5}{|l|}{ Others } \\
\hline Tetracycline & 9.3 & 26.3 & 4.8 & $\mathrm{~b}$ \\
\hline $\begin{array}{l}\text { Trimethoprim/ } \\
\text { sulfamethoxazole }\end{array}$ & 74.4 & 89.5 & 95.2 & $\mathrm{~b}$ \\
\hline Citrobacter spp. & $\begin{array}{c}\text { Bacteremia } \\
(51)\end{array}$ & $\begin{array}{c}\text { Pneumonia } \\
\text { (24) }\end{array}$ & $\begin{array}{c}\text { Bacteremia } \\
\text { (16) }\end{array}$ & $\begin{array}{c}\text { Pneumonia } \\
\left({ }^{b}\right)\end{array}$ \\
\hline \multicolumn{5}{|l|}{ Cephalosporins } \\
\hline Cefazolin & 15.7 & 33.3 & 12.5 & $\mathrm{~b}$ \\
\hline Cefuroxime & 51 & 54.2 & 50 & $\mathrm{~b}$ \\
\hline Cefoxitin & 15.7 & 33.3 & 12.5 & $\mathrm{~b}$ \\
\hline Ceftriaxone & 62.7 & 66.7 & 56.3 & $\mathrm{~b}$ \\
\hline Ceftazidime & 60.8 & 66.7 & 56.3 & $\mathrm{~b}$ \\
\hline Cefepime & 92.2 & 91.7 & 87.5 & $\mathrm{~b}$ \\
\hline \multicolumn{5}{|l|}{ Other $\beta$-lactams } \\
\hline Ampicillin & 17.6 & 12.5 & 12.5 & $\mathrm{~b}$ \\
\hline Aztreonam & 56.9 & 66.7 & 50 & $\mathrm{~b}$ \\
\hline Ticarcillin/clavulanate & 52.9 & 62.5 & 37.5 & $\mathrm{~b}$ \\
\hline Piperacillin/tazobactam & 66.7 & 75 & 62.5 & $\mathrm{~b}$ \\
\hline Imipenem & 100 & 100 & 100 & $\mathrm{~b}$ \\
\hline Meropenem & 100 & 100 & 100 & $\mathrm{~b}$ \\
\hline \multicolumn{5}{|l|}{ Aminoglycosides } \\
\hline Amikacin & 84.3 & 79.2 & 75 & $\mathrm{~b}$ \\
\hline Gentamicin & 78.4 & 83.3 & 62.5 & $\mathrm{~b}$ \\
\hline Tobramycin & 72.3 & 73.7 & 62.5 & $\mathrm{~b}$ \\
\hline \multicolumn{5}{|l|}{ Fluoroquinolones } \\
\hline Ciprofloxacin & 82.4 & 83.3 & 75 & $\mathrm{~b}$ \\
\hline Levofloxacin & 82.4 & 95.8 & 75 & $\mathrm{~b}$ \\
\hline Gatifloxacin & 80.4 & 95.8 & 75 & $\mathrm{~b}$ \\
\hline
\end{tabular}




\begin{tabular}{|c|c|c|c|c|}
\hline Garenoxacin & 83.3 & 100 & 87.5 & $\mathrm{~b}$ \\
\hline \multicolumn{5}{|l|}{ Others } \\
\hline Tetracycline & 74.5 & 70.8 & 75 & $\mathrm{~b}$ \\
\hline $\begin{array}{l}\text { Trimethoprim/ } \\
\text { sulfamethoxazole }\end{array}$ & 64.7 & 70.8 & 56.3 & $\mathrm{~b}$ \\
\hline Enterobacter spp. & $\begin{array}{c}\text { Bacteremia } \\
(517)\end{array}$ & $\begin{array}{c}\text { Pneumonia } \\
\text { (165) }\end{array}$ & $\begin{array}{c}\text { Bacteremia } \\
(266)\end{array}$ & $\begin{array}{c}\text { Pneumonia } \\
\text { (76) }\end{array}$ \\
\hline \multicolumn{5}{|l|}{ Cephalosporins } \\
\hline Cefazolin & 5.2 & 6.1 & 4.1 & 6.6 \\
\hline Cefuroxime & 37.7 & 40.6 & 44.7 & 43.4 \\
\hline Cefoxitin & 5.2 & 5.5 & 6 & 9.2 \\
\hline Ceftriaxone & 67.1 & 61.2 & 74.4 & 61.8 \\
\hline Ceftazidime & 66.9 & 63 & 73.3 & 63.2 \\
\hline Cefepime & 90.3 & 90.3 & 92.1 & 90.8 \\
\hline \multicolumn{5}{|l|}{ Other $\beta$-lactams } \\
\hline Ampicillin & 5.4 & 6.7 & 5.3 & 7.9 \\
\hline Aztreonam & 67.1 & 62.4 & 74.8 & 64.5 \\
\hline Ticarcillin/clavulanate & 55.3 & 52.7 & 61.7 & 51.3 \\
\hline Piperacillin/tazobactam & 67.9 & 64.8 & 72.9 & 65.8 \\
\hline Imipenem & 99.8 & 99.4 & 99.6 & 98.7 \\
\hline Meropenem & 99.8 & 98.8 & 99.6 & 98.7 \\
\hline \multicolumn{5}{|l|}{ Aminoglycosides } \\
\hline Amikacin & 82.6 & 86.7 & 83.1 & 86.8 \\
\hline Gentamicin & 74.5 & 79.4 & 76.7 & 78.9 \\
\hline Tobramycin & 63.8 & 68.3 & 69.6 & 69 \\
\hline \multicolumn{5}{|l|}{ Fluoroquinolones } \\
\hline Ciprofloxacin & 83.2 & 81.2 & 85.7 & 86.8 \\
\hline Levofloxacin & 84.5 & 83 & 87.6 & 89.5 \\
\hline Gatifloxacin & 85.3 & 83.6 & 89.5 & 89.5 \\
\hline Garenoxacin & 77.8 & 78.5 & 84.4 & 93.8 \\
\hline \multicolumn{5}{|l|}{ Others } \\
\hline Tetracycline & 68.3 & 73.3 & 71.1 & 71.1 \\
\hline $\begin{array}{l}\text { Trimethoprim/ } \\
\text { sulfamethoxazole }\end{array}$ & 70.7 & 77.9 & 72.5 & 76 \\
\hline E. coli & $\begin{array}{c}\text { Bacteremia } \\
(1620)\end{array}$ & $\begin{array}{c}\text { Pneumonia } \\
\text { (128) }\end{array}$ & $\begin{array}{c}\text { Bacteremia } \\
\text { (419) }\end{array}$ & $\begin{array}{c}\text { Pneumonia } \\
\text { (46) }\end{array}$ \\
\hline \multicolumn{5}{|l|}{ Cephalosporins } \\
\hline Cefazolin & 83.8 & 56.3 & 86.4 & 63 \\
\hline Cefuroxime & 88.5 & 68 & 89.5 & 69.6 \\
\hline Cefoxitin & 92.4 & 84.4 & 93.8 & 95.7 \\
\hline Ceftriaxone & $94.7(6.1)^{\mathrm{a}}$ & $78.9(21.1)^{\mathrm{a}}$ & $94.5(6)^{\mathrm{a}}$ & $76.1(19.6)^{\mathrm{a}}$ \\
\hline Ceftazidime & $95.7(6.4)^{\mathrm{a}}$ & $89.1(21.9)^{\mathrm{a}}$ & $95(6.4)^{\mathrm{a}}$ & $89.1(19.6)^{\mathrm{a}}$ \\
\hline
\end{tabular}




\begin{tabular}{|c|c|c|c|c|}
\hline Cefepime & 97.2 & 85.9 & 97.1 & 87 \\
\hline \multicolumn{5}{|l|}{ Other $\beta$-lactams } \\
\hline Ampicillin & 39.8 & 28.9 & 42.2 & 26.1 \\
\hline Aztreonam & $94.9(6.2)^{\mathrm{a}}$ & $78.7(21.3)^{\mathrm{a}}$ & $94.7(5.7)^{\mathrm{a}}$ & $80(20)^{\mathrm{a}}$ \\
\hline Ticarcillin/clavulanate & 66.7 & 48.4 & 72.8 & 58.7 \\
\hline Piperacillin/tazobactam & 92.5 & 77.3 & 93.6 & 82.6 \\
\hline Imipenem & 100 & 99.2 & 100 & 100 \\
\hline Meropenem & 100 & 100 & 100 & 100 \\
\hline \multicolumn{5}{|l|}{ Aminoglycosides } \\
\hline Amikacin & 97 & 92.2 & 97.6 & 97.8 \\
\hline Gentamicin & 90.4 & 77.3 & 93.3 & 82.6 \\
\hline Tobramycin & 88.7 & 72 & 91.5 & 73.3 \\
\hline \multicolumn{5}{|l|}{ Fluoroquinolones } \\
\hline Ciprofloxacin & 85.5 & 78.1 & 92.4 & 91.3 \\
\hline Gatifloxacin & 86.2 & 78.9 & 93.3 & 91.3 \\
\hline Levofloxacin & 86.1 & 78.1 & 93.3 & 91.3 \\
\hline Garenoxacin & 85.9 & 73.2 & 92.4 & 89.3 \\
\hline \multicolumn{5}{|l|}{ Others } \\
\hline Tetracycline & 53.7 & 54.7 & 60.9 & 65.2 \\
\hline $\begin{array}{l}\text { Trimethoprim/ } \\
\text { sulfamethoxazole }\end{array}$ & 50.9 & 58.3 & 47.5 & 63 \\
\hline K. pneumoniae & $\begin{array}{c}\text { Bacteremia } \\
(840)\end{array}$ & $\begin{array}{c}\text { Pneumonia } \\
\text { (328) }\end{array}$ & $\begin{array}{c}\text { Bacteremia } \\
\text { (328) }\end{array}$ & $\begin{array}{c}\text { Pneumonia } \\
(124)\end{array}$ \\
\hline \multicolumn{5}{|l|}{ Cephalosporins } \\
\hline Cefazolin & 47.5 & & 54.6 & 47.345 .2 \\
\hline Cefuroxime & 52.7 & 55.2 & 53.7 & 46 \\
\hline Cefoxitin & 84.6 & 82 & 86.6 & 83.9 \\
\hline Ceftriaxone & $60.7(45)^{b}$ & $63.1(41.2)^{\mathrm{b}}$ & $59.8(45.4)^{\mathrm{b}}$ & $50.8(51.6)^{\mathrm{a}}$ \\
\hline Ceftazidime & $65.2(45)^{\mathrm{b}}$ & $67.4(39.3)^{\mathrm{b}}$ & $70.1(43.9)^{\mathrm{b}}$ & $57.3(60)^{\mathrm{b}}$ \\
\hline Cefepime & 75.7 & 77.4 & 73.8 & 72.6 \\
\hline \multicolumn{5}{|l|}{ Other $\beta$-lactams } \\
\hline Ampicillin & 0 & 0 & 0 & 0 \\
\hline Aztreonam & $60.2(44)^{\mathrm{b}}$ & $63.4(40.2)^{\mathrm{b}}$ & $60.4(43.6)^{\mathrm{b}}$ & $51.6(60)^{\mathrm{b}}$ \\
\hline Ticarcillin/clavulanate & 48.9 & 56.7 & 50.9 & 46.8 \\
\hline Piperacillin/tazobactam & 65.4 & 72.3 & 69.8 & 66.9 \\
\hline Imipenem & 99.9 & 99.7 & 100 & 100 \\
\hline Meropenem & 99.8 & 99.4 & 99.7 & 99.2 \\
\hline \multicolumn{5}{|l|}{ Aminoglycosides } \\
\hline Amikacin & 78.6 & 85.7 & 80.8 & 85.5 \\
\hline Gentamicin & 62.5 & 71.6 & 63.1 & 66.9 \\
\hline Tobramycin & 50.6 & 60.6 & 52.3 & 52.7 \\
\hline \multicolumn{5}{|l|}{ Fluoroquinolones } \\
\hline Ciprofloxacin & 88.1 & 87.5 & 91.8 & 88.7 \\
\hline
\end{tabular}




\begin{tabular}{|c|c|c|c|c|}
\hline Levofloxacin & 90.1 & 88.4 & 91.8 & 88.7 \\
\hline Gatifloxacin & 91.2 & 89.9 & 93 & 88.7 \\
\hline Garenoxacin & 89.1 & 88.5 & 89.6 & 86.7 \\
\hline \multicolumn{5}{|l|}{ Others } \\
\hline Tetracycline & 65.3 & 71.6 & 72.9 & 66.1 \\
\hline $\begin{array}{l}\text { Trimethoprim/ } \\
\text { sulfamethoxazole }\end{array}$ & 66.1 & 76.1 & 59.3 & 66.1 \\
\hline Morganella morgannii & $\begin{array}{c}\text { Bacteremia } \\
\text { (32) }\end{array}$ & $\begin{array}{c}\text { Pneumonia } \\
\left({ }^{b}\right)\end{array}$ & $\begin{array}{c}\text { Bacteremia } \\
\text { (12) }\end{array}$ & $\begin{array}{c}\text { Pneumonia } \\
\left({ }^{b}\right)\end{array}$ \\
\hline \multicolumn{5}{|l|}{ Cephalosporins } \\
\hline Cefazolin & 0 & $\mathrm{~b}$ & 0 & $\mathrm{~b}$ \\
\hline Cefuroxime & 6.3 & $\mathrm{~b}$ & 8.3 & $\mathrm{~b}$ \\
\hline Cefoxitin & 31.3 & $\mathrm{~b}$ & 33.3 & $\mathrm{~b}$ \\
\hline Ceftriaxone & 87.5 & $\mathrm{~b}$ & 75 & $\mathrm{~b}$ \\
\hline Ceftazidime & 84.4 & $\mathrm{~b}$ & 91.7 & $\mathrm{~b}$ \\
\hline Cefepime & 90.6 & $\mathrm{~b}$ & 83.3 & $\mathrm{~b}$ \\
\hline \multicolumn{5}{|l|}{ Other $\beta$-lactams } \\
\hline Ampicillin & 0 & $\mathrm{~b}$ & 0 & $\mathrm{~b}$ \\
\hline Aztreonam & 84.4 & $\mathrm{~b}$ & 83.3 & $\mathrm{~b}$ \\
\hline Ticarcillin/clavulanate & 71.9 & $\mathrm{~b}$ & 83.3 & $\mathrm{~b}$ \\
\hline Piperacillin/tazobactam & 90.6 & $\mathrm{~b}$ & 100 & $\mathrm{~b}$ \\
\hline Imipenem & 100 & $\mathrm{~b}$ & 100 & $\mathrm{~b}$ \\
\hline Meropenem & 100 & $\mathrm{~b}$ & 100 & $\mathrm{~b}$ \\
\hline \multicolumn{5}{|l|}{ Aminoglycosides } \\
\hline Amikacin & 96.9 & $\mathrm{~b}$ & 91.7 & $\mathrm{~b}$ \\
\hline Gentamicin & 81.3 & $\mathrm{~b}$ & 91.7 & $\mathrm{~b}$ \\
\hline Tobramycin & 85.7 & $\mathrm{~b}$ & 81.8 & $\mathrm{~b}$ \\
\hline \multicolumn{5}{|l|}{ Fluoroquinolones } \\
\hline Ciprofloxacin & 68.8 & $\mathrm{~b}$ & 66.7 & $\mathrm{~b}$ \\
\hline Levofloxacin & 68.8 & $\mathrm{~b}$ & 66.7 & $\mathrm{~b}$ \\
\hline Gatifloxacin & 68.8 & $\mathrm{~b}$ & 66.7 & $\mathrm{~b}$ \\
\hline Garenoxacin & 66.7 & $\mathrm{~b}$ & $\mathrm{~b}$ & $\mathrm{~b}$ \\
\hline \multicolumn{5}{|l|}{ Others } \\
\hline Tetracycline & 40.6 & $\mathrm{~b}$ & 25 & $\mathrm{~b}$ \\
\hline $\begin{array}{l}\text { Trimethoprim/ } \\
\text { sulfamethoxazole }\end{array}$ & 56.3 & b & 50 & $\mathrm{~b}$ \\
\hline P. aeruginosa & $\begin{array}{c}\text { Bacteremia } \\
(589)\end{array}$ & $\begin{array}{c}\text { Pneumonia } \\
\text { (862) }\end{array}$ & $\begin{array}{c}\text { Bacteremia } \\
(247)\end{array}$ & $\begin{array}{c}\text { Pneumonia } \\
(473)\end{array}$ \\
\hline \multicolumn{5}{|l|}{ Cephalosporins } \\
\hline Ceftazidime & 69.1 & 58.7 & 66.8 & 51.8 \\
\hline $\begin{array}{c}\text { Cefepime } \\
\text { Other } \beta \text {-lactams }\end{array}$ & 71 & 58.5 & 65.2 & 51.2 \\
\hline
\end{tabular}




\begin{tabular}{|c|c|c|c|c|}
\hline Aztreonam & 51.3 & 42.1 & 49.8 & 35.1 \\
\hline Ticarcillin/clavulanate & 62.8 & 52.8 & 18.2 & 13.1 \\
\hline Piperacillin/tazobactam & 78.9 & 67.1 & 59.1 & 44 \\
\hline Imipenem & 83.4 & 65.5 & 79.8 & 56.4 \\
\hline Meropenem & 84.6 & 68.3 & 80.6 & 59.6 \\
\hline \multicolumn{5}{|l|}{ Aminoglycosides } \\
\hline Amikacin & 75.4 & 68.7 & 69.2 & 57.3 \\
\hline Gentamicin & 65.5 & 56.3 & 63.2 & 47.8 \\
\hline Tobramycin & 64.8 & 57.7 & 59 & 47.9 \\
\hline \multicolumn{5}{|l|}{ Fluoroquinolones } \\
\hline Ciprofloxacin & 66.2 & 55.7 & 66.4 & 50.7 \\
\hline Levofloxacin & 65.7 & 54.4 & 66.8 & 50.5 \\
\hline Gatifloxacin & 63.7 & 50.9 & 64.8 & 47.4 \\
\hline Garenoxacin & 57.7 & 40.7 & 58.9 & 39 \\
\hline \multicolumn{5}{|l|}{ Others } \\
\hline Tetracycline & 1.5 & 1.5 & 1.6 & 0.8 \\
\hline $\begin{array}{l}\text { Trimethoprim/ } \\
\text { sulfamethoxazole }\end{array}$ & 2.2 & 5.2 & 3.6 & 5.1 \\
\hline Proteus spp. & $\begin{array}{c}\text { Bacteremia } \\
(92)\end{array}$ & $\begin{array}{c}\text { Pneumonia } \\
\text { (42) }\end{array}$ & $\begin{array}{c}\text { Bacteremia } \\
\text { (15) }\end{array}$ & $\begin{array}{c}\text { Pneumonia } \\
\left(\left(^{b}\right)\right.\end{array}$ \\
\hline \multicolumn{5}{|l|}{ Cephalosporins } \\
\hline Cefazolin & 54.3 & 54.8 & 80 & $\mathrm{~b}$ \\
\hline Cefuroxime & 65.2 & 59.5 & 86.7 & $\mathrm{~b}$ \\
\hline Cefoxitin & 95.7 & 90.5 & 100 & $\mathrm{~b}$ \\
\hline Ceftriaxone & 72.8 & 66.7 & 100 & $\mathrm{~b}$ \\
\hline Ceftazidime & 93.5 & 97.6 & 100 & $\mathrm{~b}$ \\
\hline Cefepime & 75 & 66.7 & 100 & $\mathrm{~b}$ \\
\hline \multicolumn{5}{|l|}{ Other $\beta$-lactams } \\
\hline Ampicillin & 43.5 & 57.1 & 60 & $\mathrm{~b}$ \\
\hline Aztreonam & 92.4 & 83.3 & 100 & $\mathrm{~b}$ \\
\hline Ticarcillin/clavulanate & 90.2 & 97.6 & 100 & $\mathrm{~b}$ \\
\hline Piperacillin/tazobactam & 91.3 & 92.9 & 100 & $\mathrm{~b}$ \\
\hline Imipenem & 97.8 & 100 & 93.3 & $\mathrm{~b}$ \\
\hline Meropenem & 100 & 100 & 100 & $\mathrm{~b}$ \\
\hline \multicolumn{5}{|l|}{ Aminoglycosides } \\
\hline Amikacin & 91.3 & 88.1 & 100 & $\mathrm{~b}$ \\
\hline Gentamicin & 63 & 69 & 100 & $\mathrm{~b}$ \\
\hline Tobramycin & 65.4 & 69.4 & 100 & $\mathrm{~b}$ \\
\hline \multicolumn{5}{|l|}{ Fluoroquinolones } \\
\hline Ciprofloxacin & 68.5 & 71.4 & 100 & $\mathrm{~b}$ \\
\hline Levofloxacin & 70.7 & 76.2 & 100 & $\mathrm{~b}$ \\
\hline Gatifloxacin & 67.4 & 71.4 & 100 & $\mathrm{~b}$ \\
\hline Garenoxacin & 70.2 & 72 & $\mathrm{~b}$ & $\mathrm{~b}$ \\
\hline
\end{tabular}


Others

Tetracycline

4.3

7.1

13.3

b

Trimethoprim/ sulfamethoxazole
53.3
64.3
86.7

Salmonella spp.

Bacteremia

(179)

(b)

(44)

100

97.8

95.5

98.3

98.9

98.9

98.9

Cefepime

Other $\beta$-lactams

Ampicillin

Aztreonam

Ticarcillin/clavulanate

Piperacillin/tazobactam

Imipenem

Meropenem

Aminoglycosides

Amikacin

Gentamicin

Tobramycin

Fluoroquinolones

Ciprofloxacin

Levofloxacin

Gatifloxacin

Garenoxacin

Others

Tetracycline

Trimethoprim/

sulfamethoxazole

Serratia spp.

Cephalosporins

Cefazolin

Cefuroxime

Cefoxitin

Ceftriaxone

Ceftazidime

Cefepime

Other $\beta$-lactams
92.2

98.3

93.3

98.9

100

100

99.4

96.1

94.7

100

100

100

100

87.2

93.3

Bacteremia

(157)

\section{Pneumonia}

(89)

100

100

100

100

97.7

100

100

100

100

100

100

100

100

95.5

91.7

Bacteremia

(86)
100

100

100

0

2.2

3.4

3.4

8.3

80.9

72.6

85.4

93.3

97.7

(b)

Pneumonia

95.5

b

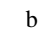

b

b

b

b

b

b

b

b

b

b

Pneumonia

(40)

$\begin{array}{cc}0 & 0 \\ 0 & 0 \\ 5.8 & 2.5 \\ 69.8 & 72.5 \\ 81.4 & 77.5 \\ 90.7 & 97.5\end{array}$




\begin{tabular}{|c|c|c|c|c|}
\hline Ampicillin & 4.5 & 1.1 & 2.3 & 0 \\
\hline Aztreonam & 79.6 & 88.8 & 82.6 & 87.5 \\
\hline Ticarcillin/clavulanate & 57.3 & 60.7 & 48.8 & 45 \\
\hline Piperacillin/tazobactam & 72 & 77.5 & 62.8 & 70 \\
\hline Imipenem & 100 & 98.9 & 100 & 100 \\
\hline Meropenem & 100 & 100 & 100 & 100 \\
\hline \multicolumn{5}{|l|}{ Aminoglycosides } \\
\hline Amikacin & 70.7 & 80.9 & 64 & 75 \\
\hline Gentamicin & 63.7 & 75.3 & 53.5 & 62.5 \\
\hline Tobramycin & 51.8 & 57.9 & 42.9 & 45.9 \\
\hline \multicolumn{5}{|l|}{ Fluoroquinolones } \\
\hline Ciprofloxacin & 75.2 & 78.7 & 60.5 & 67.5 \\
\hline Levofloxacin & 82.2 & 87.6 & 69.8 & 77.5 \\
\hline Gatifloxacin & 79.6 & 84.3 & 65.1 & 75 \\
\hline Garenoxacin & 59.2 & 75 & 46.5 & 63.2 \\
\hline \multicolumn{5}{|l|}{ Others } \\
\hline Tetracycline & 9.6 & 7.9 & 4.7 & 5 \\
\hline $\begin{array}{l}\text { Trimethoprim/ } \\
\text { sulfamethoxazole }\end{array}$ & 63.1 & 70.8 & 54.7 & 60 \\
\hline Stenotrophomonas maltophilia & $\begin{array}{c}\text { Bacteremia } \\
(\mathbf{8 6})\end{array}$ & $\begin{array}{c}\text { Pneumonia } \\
\qquad(60)\end{array}$ & $\begin{array}{c}\text { Bacteremia } \\
\text { (35) }\end{array}$ & $\begin{array}{c}\text { Pneumonia } \\
(23)\end{array}$ \\
\hline \multicolumn{5}{|l|}{ Cephalosporins } \\
\hline Ceftazidime & 73.3 & 46.7 & 85.7 & 47.8 \\
\hline Cefepime & 40.7 & 23.3 & 42.9 & 30.4 \\
\hline \multicolumn{5}{|l|}{ Other $\beta$-lactams } \\
\hline Aztreonam & 8.1 & 1.7 & 8.6 & 0 \\
\hline Ticarcillin/clavulanate & 64 & 51.7 & 77.1 & 69.6 \\
\hline Piperacillin/tazobactam & 43.5 & 26.7 & 51.4 & 30.4 \\
\hline Imipenem & 2.3 & 0 & 0 & 0 \\
\hline Meropenem & 7 & 3.3 & 5.7 & 8.7 \\
\hline \multicolumn{5}{|l|}{ Aminoglycosides } \\
\hline Amikacin & 20.9 & 15 & 17.1 & 8.7 \\
\hline Gentamicin & 20.9 & 11.7 & 14.3 & 8.7 \\
\hline Tobramycin & 19 & 8.9 & 8.6 & 8.7 \\
\hline \multicolumn{5}{|l|}{ Fluoroquinolones } \\
\hline Ciprofloxacin & 50 & 38.3 & 45.7 & 17.4 \\
\hline Levofloxacin & 88.4 & 90 & 94.3 & 87 \\
\hline Gatifloxacin & 88.4 & 96.7 & 94.3 & 95.7 \\
\hline Garenoxacin & 83.3 & 81.8 & 79.2 & 80 \\
\hline \multicolumn{5}{|l|}{ Others } \\
\hline Tetracycline & 17.4 & 13.3 & 17.1 & 8.7 \\
\hline $\begin{array}{l}\text { Trimethoprim/ } \\
\text { sulfamethoxazole }\end{array}$ & 95.3 & 100 & 100 & 100 \\
\hline
\end{tabular}

a. Percentage of strains with MIC $\geq 2 \mu \mathrm{g} / \mathrm{mL}$, indicating possible ESBL production [6]; b. The results were not analyzed because the number of isolates was low $(<10)$. 
Table 9. Antimicrobial activity and spectrum of drugs tested against the most prevalent Gram-positive pathogens isolated from January 1997 to December 2001 throughout Latin America and in Brazil alone, from patients with bacteremia and pneumonia

\begin{tabular}{|c|c|c|c|c|}
\hline \multirow{3}{*}{$\begin{array}{l}\text { Pathogen/ } \\
\text { Antimicrobial agent } \\
\text { S. aureus }\end{array}$} & \multicolumn{4}{|c|}{ \% Susceptible strains (number of isolates tested) } \\
\hline & \multicolumn{2}{|c|}{ Latin America } & \multicolumn{2}{|c|}{ Brazil } \\
\hline & $\begin{array}{c}\text { Bacteremia } \\
(\mathbf{1 , 9 2 1 )}\end{array}$ & $\begin{array}{c}\text { Pneumonia } \\
(\mathbf{7 6 0})\end{array}$ & $\begin{array}{c}\text { Bacteremia } \\
\qquad(840)\end{array}$ & $\begin{array}{c}\text { Pneumonia } \\
\text { (346) }\end{array}$ \\
\hline \multicolumn{5}{|l|}{ Cephalosporins } \\
\hline Cefazolin & $68.5^{\mathrm{a}}$ & $45.4^{\mathrm{a}}$ & $68.2^{\mathrm{a}}$ & $48.8^{\mathrm{a}}$ \\
\hline Ceftriaxone & $68.5^{\mathrm{a}}$ & $45.4^{\mathrm{a}}$ & $68.2^{\mathrm{a}}$ & $48.8^{\mathrm{a}}$ \\
\hline Cefepime & $68.5^{\mathrm{a}}$ & $45.4^{\mathrm{a}}$ & $68.2^{\mathrm{a}}$ & $48.8^{\mathrm{a}}$ \\
\hline \multicolumn{5}{|l|}{ Other $\beta$-lactams } \\
\hline Oxacillin & 68.5 & 45.4 & 68.2 & 48.8 \\
\hline Penicillin & 8.5 & 5.7 & 11.2 & 6.4 \\
\hline Amoxicillin/clavulanate & $68.5^{\mathrm{a}}$ & $45.4^{\mathrm{a}}$ & $68.2^{\mathrm{a}}$ & $48.8^{\mathrm{a}}$ \\
\hline Piperacillin/tazobactam & $68.5^{\mathrm{a}}$ & $45.4^{\mathrm{a}}$ & $68.2^{\mathrm{a}}$ & $48.8^{\mathrm{a}}$ \\
\hline Imipenem & $68.5^{\mathrm{a}}$ & $45.4^{\mathrm{a}}$ & $68.2^{\mathrm{a}}$ & $48.8^{\mathrm{a}}$ \\
\hline \multicolumn{5}{|l|}{ MLS } \\
\hline Clindamycin & 72 & 46.3 & 69.9 & 48.6 \\
\hline Erythromycin & 54.6 & 37.4 & 52.5 & 38.4 \\
\hline \multicolumn{5}{|l|}{ Fluoroquinolones } \\
\hline Ciprofloxacin & 70.3 & 45.9 & 69.5 & 48 \\
\hline Levofloxacin & 72.9 & 48.4 & 72.2 & 50.3 \\
\hline Gatifloxacin & 90.7 & 85.7 & 88.3 & 85.3 \\
\hline Garenoxacin & 97.9 & 95.4 & 97.1 & 95.1 \\
\hline \multicolumn{5}{|l|}{ Others } \\
\hline Gentamicin & 67.4 & 45.8 & 66.8 & 49.1 \\
\hline Rifampin & 80.4 & 66.3 & 74.8 & 53.8 \\
\hline Chloramphenicol & 68 & 54.5 & 68.6 & 54.3 \\
\hline Tetracycline & 75 & 61.6 & 66.3 & 48.6 \\
\hline Doxycycline & 87 & 75.7 & 80.1 & 64.7 \\
\hline Trimetoprim/sulfametoxale & 79.4 & 64.8 & 87 & 74.8 \\
\hline Vancomycin & 100 & 100 & 100 & 100 \\
\hline Teicoplanin & 99.5 & 99.9 & 99.3 & 100 \\
\hline Quinupristin/dalfopristin & 99.6 & 100 & 99.8 & 100 \\
\hline Linezolid & 100 & 100 & 100 & 100 \\
\hline CoNS & $\begin{array}{c}\text { Bacteremia } \\
(\mathbf{1}, 221)\end{array}$ & $\begin{array}{c}\text { Pneumonia } \\
\text { (41) }\end{array}$ & $\begin{array}{c}\text { Bacteremia } \\
(\mathbf{4 5 8})\end{array}$ & $\begin{array}{c}\text { Pneumonia } \\
\text { (19) }\end{array}$ \\
\hline \multicolumn{5}{|l|}{ Cephalosporins } \\
\hline Cefazolin & $23.8^{\mathrm{a}}$ & $24.4^{\mathrm{a}}$ & $19.2^{\mathrm{a}}$ & $36.8^{\mathrm{a}}$ \\
\hline Ceftriaxone & $23.8^{\mathrm{a}}$ & $24.4^{\mathrm{a}}$ & $19.2^{\mathrm{a}}$ & $36.8^{\mathrm{a}}$ \\
\hline
\end{tabular}




\begin{tabular}{|c|c|c|c|c|}
\hline Cefepime & $23.8^{\mathrm{a}}$ & $24.4^{\mathrm{a}}$ & $19.2^{\mathrm{a}}$ & $36.8^{\mathrm{a}}$ \\
\hline \multicolumn{5}{|l|}{ Other $\beta$-lactams } \\
\hline Oxacillin & 23.8 & 24.4 & 19.2 & 36.8 \\
\hline Penicillin & 9.3 & 12.2 & 7.9 & 21.1 \\
\hline Amoxicillin/clavulanate & $23.8^{\mathrm{a}}$ & $24.4^{\mathrm{a}}$ & $19.2^{\mathrm{a}}$ & $36.8^{\mathrm{a}}$ \\
\hline Piperacillin/tazobactam & $23.8^{\mathrm{a}}$ & $24.4^{\mathrm{a}}$ & $19.2^{\mathrm{a}}$ & $36.8^{\mathrm{a}}$ \\
\hline Imipenem & $23.8^{\mathrm{a}}$ & $24.4^{\mathrm{a}}$ & $19.2^{\mathrm{a}}$ & $36.8^{\mathrm{a}}$ \\
\hline \multicolumn{5}{|l|}{ MLS } \\
\hline Clindamycin & 55.8 & 36.6 & 46.3 & 47.4 \\
\hline Erythromycin & 36 & 17.1 & 35.4 & 26.3 \\
\hline \multicolumn{5}{|l|}{ Fluoroquinolones } \\
\hline Ciprofloxacin & 58 & 31.7 & 48.5 & 42.1 \\
\hline Levofloxacin & 66.2 & 57.9 & 59.9 & 53.3 \\
\hline Gatifloxacin & 94.6 & 80.5 & 93 & 84.2 \\
\hline Garenoxacin & 97.9 & 67.9 & 92.6 & 87.5 \\
\hline \multicolumn{5}{|l|}{ Others } \\
\hline Gentamicin & 44.8 & 36.6 & 40 & 42.1 \\
\hline Rifampin & 72.5 & 68.3 & 69 & 63.2 \\
\hline Chloramphenicol & 62.2 & 63.4 & 55.2 & 68.4 \\
\hline Tetracycline & 77.1 & 75.6 & 75.8 & 57.9 \\
\hline Doxycycline & 87.7 & 82.9 & 86.7 & 68.4 \\
\hline Trimethoprim/sulfamethoxale & e $\quad 51.1$ & 70.7 & 69.8 & 68.4 \\
\hline Vancomycin & 100 & 100 & 100 & 100 \\
\hline Teicoplanin & 90.6 & 85.4 & 90.4 & 89.5 \\
\hline Quinupristin/dalfopristin & 98.6 & 100 & 98 & 100 \\
\hline Linezolid & 100 & 100 & 100 & 100 \\
\hline Enterococcus spp. & $\begin{array}{c}\text { Bacteremia } \\
\text { (280) }\end{array}$ & $\begin{array}{c}\text { Pneumonia } \\
\text { (58) }\end{array}$ & $\begin{array}{c}\text { Bacteremia } \\
(\mathbf{1 0 1})\end{array}$ & $\begin{array}{c}\text { Pneumonia } \\
(44)\end{array}$ \\
\hline \multicolumn{5}{|l|}{$\beta$-lactams } \\
\hline Ampicillin & 91.8 & 100 & 95 & 100 \\
\hline Penicillin & 84.6 & 81 & 78.2 & 75 \\
\hline Amoxicillin/Clavulanate & $91.8^{b}$ & $100^{\mathrm{b}}$ & $95^{\mathrm{b}}$ & $100^{\mathrm{b}}$ \\
\hline Piperacillin/Tazobactam & $91.8^{b}$ & $100^{\mathrm{b}}$ & $95^{b}$ & $100^{\mathrm{b}}$ \\
\hline \multicolumn{5}{|l|}{ MLS } \\
\hline Erythromycin & 9.3 & 13.8 & 5 & 15.9 \\
\hline \multicolumn{5}{|l|}{ Fluoroquinolones } \\
\hline Ciprofloxacin & 48.2 & 48.3 & 40.6 & 43.2 \\
\hline Levofloxacin & 67.2 & 41.2 & 57.4 & 36.4 \\
\hline Gatifloxacin & 72.1 & 63.8 & 62.4 & 63.6 \\
\hline Garenoxacin & 90 & 91.2 & 90.6 & 91.3 \\
\hline \multicolumn{5}{|l|}{ Others } \\
\hline Gentamicin (HL) & 73.9 & 65.5 & 57.4 & 65.9 \\
\hline Streptomycin (HL) & 88.2 & 91.4 & 92.1 & 95.5 \\
\hline
\end{tabular}




\begin{tabular}{lllll}
\hline Rifampin & 25.4 & 20.7 & 19.8 & 11.4 \\
Chloramphenicol & 66.4 & 55.2 & 50.5 & 54.5 \\
Tetracycline & 36.1 & 37.9 & 35.6 & 36.4 \\
Doxycycline & 47.5 & 48.3 & 53.5 & 50 \\
Vancomycin & 97.4 & 100 & 98 & 100 \\
Teicoplanin & 97.5 & 100 & 98 & 100 \\
Quinupristin/dalfopristin & 12.1 & 3.4 & 7.9 & 4.5 \\
Linezolid & 96.1 & 97.9 & 97.5 & 97.1 \\
\hline
\end{tabular}

a. Interpreted by NCCLS 2003 criteria, when available [6]; b. Susceptibility is predicted by the oxacillin result [6]; c. Susceptibility is predicted by the ampicillin result [6]. Abbreviation: HL - High level aminoglycoside resistance screen.

Table 10. Antimicrobial activity and spectrum of drugs tested against the most prevalent streptococci isolated from January 1997 to December 2001 throughout Latin America and in Brazil alone

\begin{tabular}{|c|c|c|c|c|}
\hline \multirow{2}{*}{$\begin{array}{l}\text { Pathogen (no total/Brazil)/ } \\
\text { Antimicrobial Agent }\end{array}$} & \multicolumn{2}{|c|}{ Latin America $^{a}$} & \multicolumn{2}{|c|}{ Brazil $^{\mathbf{a}}$} \\
\hline & $\mathrm{MIC}_{50}$ & $\mathrm{MIC}_{90}$ & \% susceptible & $\%$ susceptible \\
\hline \multicolumn{5}{|l|}{$\begin{array}{l}\beta \text {-haemolytic streptococci } \\
(443 / 178)\end{array}$} \\
\hline Amoxicillin & 0.06 & 0.12 & $100^{\mathrm{d}}$ & $100^{\mathrm{d}}$ \\
\hline Penicillin & 0.03 & 0.06 & 100 & 100 \\
\hline Cefuroxime $^{b}$ & 0.06 & 0.12 & $100^{\mathrm{d}}$ & $100^{\mathrm{d}}$ \\
\hline Cefprozil & $\leq 0.12$ & 0.5 & $100^{\mathrm{d}}$ & $100^{\mathrm{d}}$ \\
\hline Cefpodoxime & 0.06 & 0.12 & $100^{\mathrm{d}}$ & $100^{\mathrm{d}}$ \\
\hline Cefotaxime $^{c}$ & 0.06 & 0.12 & 100 & 100 \\
\hline Cefepime & 0.12 & 0.12 & 100 & 100 \\
\hline Erythromycin & 0.06 & 0.25 & 92.8 & 96.6 \\
\hline Azithromycin & 0.06 & 0.12 & 92.2 & 95 \\
\hline Clarithromycin & 0.25 & 0.25 & 96.1 & 94.1 \\
\hline Clindamycin & 0.06 & 0.12 & 97.7 & 99.4 \\
\hline Levofloxacin & 0.5 & 1 & 100 & 100 \\
\hline Gatifloxacin & 0.25 & 0.5 & 100 & 100 \\
\hline Garenoxacin & 0.06 & 0.12 & -- & -- \\
\hline Chloramphenicol & $\leq 2$ & 4 & 98.4 & 100 \\
\hline Tetracycline $^{e}$ & 4 & $>8$ & 49.9 & 38.8 \\
\hline Trimethoprim/sulfamethoxale & $\leq 0.5$ & $\leq 0.5$ & NA & NA \\
\hline Linezolid & 1 & 1 & 100 & 100 \\
\hline Quinupristin/dalfopristin & 0.25 & 0.5 & 100 & 100 \\
\hline Vancomycin & 0.5 & 0.5 & 100 & 100 \\
\hline \multicolumn{5}{|l|}{ S. bovis $(29 / 18)$} \\
\hline Amoxicillin & 0.06 & 0.12 & $100^{\mathrm{d}}$ & $94.4^{\mathrm{d}}$ \\
\hline
\end{tabular}




\begin{tabular}{|c|c|c|c|c|}
\hline Penicillin & 0.06 & 0.12 & 100 & 94.4 \\
\hline Cefuroxime $^{b}$ & 0.12 & 0.25 & $100^{\mathrm{d}}$ & $94.4^{\mathrm{d}}$ \\
\hline Cefprozil & 0.5 & 1 & $100^{\mathrm{d}}$ & $94.4^{\mathrm{d}}$ \\
\hline Cefpodoxime & 0.25 & 0.5 & $100^{\mathrm{d}}$ & $94.4^{\mathrm{d}}$ \\
\hline Cefotaxime $^{\mathrm{c}}$ & 0.12 & 0.25 & 100 & 100 \\
\hline Cefepime & 0.12 & 0.25 & 96.6 & 94.4 \\
\hline Erythromycin & 0.25 & $>8$ & 62.1 & 61.1 \\
\hline Azithromycin & 0.12 & $>16$ & 66.7 & 60 \\
\hline Clarithromycin & 0.25 & $>32$ & 75 & 62.5 \\
\hline Clindamycin & 0.12 & $>8$ & 72.4 & 72.2 \\
\hline Levofloxacin & 1 & $>2$ & 100 & 100 \\
\hline Gatifloxacin & 0.5 & 0.5 & 100 & 100 \\
\hline Garenoxacin & 0.06 & 0.12 & - & - \\
\hline Chloramphenicol & 4 & 4 & 100 & 100 \\
\hline Tetracycline $^{\mathrm{e}}$ & $>8$ & $>8$ & 48.3 & 33.3 \\
\hline Trimethoprim/sulfamethoxale & $\leq 0.5$ & $>1$ & NA & NA \\
\hline Linezolid & 1 & 2 & 100 & 100 \\
\hline Quinupristin/dalfopristin & 1 & 4 & 86.2 & 83.3 \\
\hline Vancomycin & 0.5 & 0.5 & 100 & 100 \\
\hline \multicolumn{5}{|l|}{ S. pneumoniae (1561/497) } \\
\hline Amoxicillin & 0.06 & 1 & 98.2 & 98.8 \\
\hline Penicillin & 0.03 & 2 & $69.3(11.9)^{\mathrm{f}}$ & $78.5(4.6)^{f}$ \\
\hline Cefuroxime $^{\mathrm{b}}$ & 0.06 & 4 & 84.5 & 93.5 \\
\hline Cefprozil & 0.25 & 8 & 84.1 & 93.1 \\
\hline Cefpodoxime & $\leq 0.03$ & 2 & 84.4 & 93.8 \\
\hline Cefotaxime $^{c}$ & 0.03 & 0.5 & 98.3 & 98.6 \\
\hline Cefepime & 0.06 & 1 & 97.5 & 99.2 \\
\hline Erythromycin & 0.25 & 2 & 87.1 & 88.5 \\
\hline Azithromycin & 0.12 & 2 & 88.5 & 91.4 \\
\hline Clarithromycin & 0.25 & 1 & 87.5 & 89.2 \\
\hline Clindamycin & 0.06 & 0.25 & 94.2 & 94.6 \\
\hline Levofloxacin & 1 & 1 & 99.8 & 100 \\
\hline Gatifloxacin & 0.25 & 0.5 & 99.7 & 100 \\
\hline Garenoxacin & 0.06 & 0.06 & - & - \\
\hline Chloramphenicol & $\leq 2$ & 4 & 95.6 & 98.2 \\
\hline Tetracycline $^{\mathrm{e}}$ & $\leq 2$ & $>16$ & 79.5 & 80.5 \\
\hline Trimethoprim/sulfamethoxale & $\leq 0.5$ & 4 & 60.5 & 49.7 \\
\hline Linezolid & 1 & 1 & 100 & 100 \\
\hline Quinupristin/dalfopristin & 0.5 & 0.5 & 100 & 100 \\
\hline Vancomycin & 0.25 & 0.5 & 100 & 100 \\
\hline \multicolumn{5}{|l|}{ Viridans group $(124 / 36)$} \\
\hline Amoxicillin & 0.12 & 4 & 66.1 & 80.6 \\
\hline
\end{tabular}




\begin{tabular}{lcccc}
\hline Penicillin & 0.06 & 2 & 66.1 & 80.6 \\
Cefuroxime & 0.25 & 8 & $66.1^{\mathrm{d}}$ & 80.6 \\
Cefprozil & 1 & 8 & 66.1 & 80.6 \\
Cefpodoxime & 0.12 & $>4$ & $66.1^{\mathrm{d}}$ & 80.6 \\
Cefotaxime $^{\mathrm{c}}$ & 0.12 & 2 & 85.7 & 100 \\
Cefepime & 0.25 & 2 & 89.5 & 94.4 \\
Erythromycin & 0.25 & 2 & 65.3 & 72.2 \\
Azithromycin & 0.12 & 1 & 84.7 & 78.9 \\
Clarithromycin & 0.25 & 0.25 & 92.9 & 100 \\
Clindamycin & 0.06 & 0.12 & 91.9 & 97.2 \\
Levofloxacin & 1 & 2 & 100 & 100 \\
Gatifloxacin & 0.25 & 0.5 & 100 & 100 \\
Garenoxacin & 0.06 & 0.12 & - & - \\
Chloramphenicol & $\leq 2$ & 4 & 97.6 & 100 \\
Tetracycline & $\leq 4$ & $>16$ & 69.4 & 72.2 \\
Trimethoprim/sulfamethoxale & $\leq 0.5$ & 2 & $\mathrm{NA}$ & $\mathrm{NA}$ \\
Linezolid & 1 & 1 & 100 & 100 \\
Quinupristin/dalfopristin & 0.5 & 1 & 96 & 97.2 \\
Vancomycin & 0.5 & 1 & 100 & 100 \\
\hline
\end{tabular}

a. Interpreted by NCCLS criteria [6]; b. Percentage of susceptible strains defined by cefuroxime axetil (oral) [6]; c. Also indicates susceptibility spectrum of ceftriaxone [6]; d. Susceptibility is predicted by the penicillin result [6]; e. Includes susceptible and intermediate isolates [6]; f. Percentage of isolates showing high level resistance (MIC $\geq 2 \mu \mathrm{g} / \mathrm{ml}$ ) [6].

Table 11a. Antimicrobial activity and spectrum of drugs tested against ESBL-producing Escherichia coli and Klebsiella pneumoniae in the year 2001 throughout Latin America and in Brazil alone

\begin{tabular}{|c|c|c|}
\hline \multirow{2}{*}{$\begin{array}{l}\text { Pathogen (no total/Brazil)/ } \\
\text { Antimicrobial agent }\end{array}$} & \multirow{2}{*}{$\frac{\text { Latin America }}{\% \text { Susceptible }}$} & \multirow{2}{*}{$\frac{\text { Brazil }}{\text { \% Susceptible }}$} \\
\hline & & \\
\hline \multicolumn{3}{|l|}{ E. $\operatorname{coli}\left(35 /^{a}\right)$} \\
\hline \multicolumn{3}{|l|}{ Cephalosporins } \\
\hline Cefazolin & 20 & a \\
\hline Cefuroxime & 22.9 & a \\
\hline Cefoxitin & 54.3 & a \\
\hline Ceftriaxone & 42.9 & a \\
\hline Ceftazidime & 48.6 & a \\
\hline Cefepime & 62.9 & a \\
\hline Other $\beta$-lactams & & a \\
\hline Ampicillin & 0 & a \\
\hline Aztreonam & 42.9 & a \\
\hline Ticarcillin/clavulanate & 22.9 & a \\
\hline Piperacillin/tazobactam & 65.7 & a \\
\hline
\end{tabular}




\begin{tabular}{|c|c|c|}
\hline Imipenem & 97.1 & a \\
\hline Meropenem & 100 & a \\
\hline Aminoglycosides & & a \\
\hline Amikacin & 82.9 & a \\
\hline Gentamicin & 54.3 & a \\
\hline Tobramycin & 45.7 & a \\
\hline Fluoroquinolones & & a \\
\hline Ciprofloxacin & 42.9 & a \\
\hline Levofloxacin & 48.6 & a \\
\hline Gatifloxacin & 48.6 & a \\
\hline Garenoxacin & 40 & a \\
\hline Others & & a \\
\hline Tetracycline & 34.3 & a \\
\hline $\begin{array}{l}\text { Trimethoprim/ } \\
\text { sulfamethoxazole }\end{array}$ & 17.1 & a \\
\hline \multicolumn{3}{|c|}{ Klebsiella pneumoniae (122/63) } \\
\hline \multicolumn{3}{|c|}{ Cephalosporins } \\
\hline Cefazolin & 0.8 & 0 \\
\hline Cefuroxime & 2.5 & 1.6 \\
\hline Cefoxitin & 71.3 & 71.4 \\
\hline Ceftriaxone & 17.2 & 7.9 \\
\hline Ceftazidime & 30.3 & 22.2 \\
\hline Cefepime & 45.1 & 33.3 \\
\hline \multicolumn{3}{|l|}{ Other $\beta$-lactams } \\
\hline Ampicillin & 0 & 0 \\
\hline Aztreonam & 15.6 & 3.2 \\
\hline Ticarcillin/clavulanate & 4.9 & 3.2 \\
\hline Piperacillin/tazobactam & 46.7 & 39.7 \\
\hline Imipenem & 100 & 100 \\
\hline Meropenem & 97.5 & 96.8 \\
\hline \multicolumn{3}{|l|}{ Aminoglycosides } \\
\hline Amikacin & 63.9 & 74.6 \\
\hline Gentamicin & 24.6 & 27 \\
\hline Tobramycin & 13.1 & 12.7 \\
\hline \multicolumn{3}{|l|}{ Fluoroquinolones } \\
\hline Ciprofloxacin & 67.2 & 65.1 \\
\hline Levofloxacin & 70.5 & 65.1 \\
\hline Gatifloxacin & 70.5 & 65.1 \\
\hline Garenoxacin & 66.4 & 66.7 \\
\hline \multicolumn{3}{|l|}{ Others } \\
\hline Tetracycline & 53.3 & 55.6 \\
\hline Trimethoprim/ & & \\
\hline sulfamethoxazole & 41 & 34.9 \\
\hline
\end{tabular}


Table 11b. Antimicrobial activity and spectrum of drugs tested against ESBL-producing E. coli and $K$. pneumoniae in the year 2001 throughout Latin America from patients with bloodstream infections and lower respiratory tract infections.

\begin{tabular}{|c|c|c|c|c|}
\hline \multirow{3}{*}{$\begin{array}{l}\text { Pathogen/ } \\
\text { Antimicrobial agent } \\
\text { E. coli }\end{array}$} & \multicolumn{4}{|c|}{ \% Susceptible strains (number of isolates tested) } \\
\hline & \multicolumn{2}{|c|}{ Latin America } & \multicolumn{2}{|r|}{ Brazil } \\
\hline & $\begin{array}{c}\text { Bacteremia } \\
\text { (27) }\end{array}$ & $\begin{array}{c}\text { Pneumonia } \\
\left({ }^{a}\right)\end{array}$ & $\begin{array}{c}\text { Bacteremia } \\
\text { (a) }\end{array}$ & $\begin{array}{c}\text { Pneumonia } \\
\text { (a) }\end{array}$ \\
\hline \multicolumn{5}{|l|}{ Cephalosporins } \\
\hline Cefazolin & 22.2 & a & a & a \\
\hline Cefuroxime & 25.9 & a & a & a \\
\hline Cefoxitin & 59.3 & a & a & a \\
\hline Ceftriaxone & 48.1 & a & a & a \\
\hline Ceftazidime & 48.1 & a & a & a \\
\hline Cefepime & 66.7 & a & a & a \\
\hline Other $\beta$-lactams & & a & a & a \\
\hline Ampicillin & 0 & $\mathbf{a}$ & a & a \\
\hline Aztreonam & 51.9 & a & a & a \\
\hline Ticarcillin/clavulanate & 22.2 & $\mathbf{a}$ & a & a \\
\hline Piperacillin/tazobactam & 66.7 & a & a & a \\
\hline Imipenem & 100 & a & a & a \\
\hline Meropenem & 100 & a & a & a \\
\hline Aminoglycosides & & $\mathbf{a}$ & a & a \\
\hline Amikacin & 81.5 & a & a & a \\
\hline Gentamicin & 59.3 & a & a & a \\
\hline Tobramycin & 44.4 & a & a & a \\
\hline Fluoroquinolones & & a & a & a \\
\hline Ciprofloxacin & 40.7 & a & a & a \\
\hline Levofloxacin & 48.1 & a & a & a \\
\hline Gatifloxacin & 44.4 & a & a & a \\
\hline Garenoxacin & 37 & a & a & a \\
\hline Others & & $\mathbf{a}$ & a & a \\
\hline Tetracycline & 33.3 & a & a & a \\
\hline $\begin{array}{l}\text { Trimethoprim/ } \\
\text { sulfamethoxazole }\end{array}$ & 18.5 & a & a & a \\
\hline
\end{tabular}

\section{Klebsiella pneumoniae}

Cephalosporins

Cefazolin

Cefuroxime

Cefoxitin

Ceftriaxone
Bacteremia

(69)

(30)

0

2.9

71

14.5

\section{3}

3.3

76.7

26.7
(35)

\section{Bacteremia}

0

2.9

74.3

8.6
(13)

Pneumonia

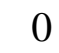

0

69.2 


\begin{tabular}{lcccc}
\hline Ceftazidime & 23.2 & 56.7 & 22.9 & 30.8 \\
Cefepime & 46.4 & 56.7 & 37.1 & 38.5 \\
Other $\beta$-lactams & & & & \\
Ampicillin & 0 & 0 & 0 & 0 \\
Aztreonam & 10.1 & 36.7 & 2.9 & 7.7 \\
Ticarcillin/clavulanate & 2.9 & 10 & 2.9 & 0 \\
Piperacillin/tazobactam & 37.7 & 66.7 & 37.1 & 53.8 \\
Imipenem & 100 & 100 & 100 & 100 \\
Meropenem & 98.6 & 100 & 97.1 & 100 \\
Aminoglycosides & & & & \\
Amikacin & 62.3 & 76.7 & 77.1 & 76.9 \\
Gentamicin & 23.2 & 36.7 & 28.6 & 30.8 \\
Tobramycin & 11.6 & 20 & 17.1 & 0 \\
Fluoroquinolones & & & & 69.2 \\
Ciprofloxacin & 66.7 & 80 & 71.4 & 69.2 \\
Levofloxacin & 71 & 83.3 & 71.4 & 69.2 \\
Gatifloxacin & 71 & 83.3 & 71.4 & 69.2 \\
Garenoxacin & 65.2 & 80 & 71.4 & 69.2 \\
Others & & & & 51.4 \\
Tetracycline & 49.3 & 73.3 & 31.4 & 53.8 \\
Trimethoprim/ & & & & \\
sulfamethoxazole & 37.7 & 60 & & \\
\hline
\end{tabular}

Table 11c. Antimicrobial activity and spectrum of drugs tested against ESBL-producing E. coli and K. pneumoniae collected throughout Latin America and in Brazil alone, from January 1997 to December 2001

\begin{tabular}{llll}
\hline Pathogen (no. total/Brazil)/ & Latin America & & Brazil \\
Antimicrobial agent & \% Susceptible & & \% Susceptible \\
\hline E. coli (250/64) & & \\
Cephalosporins & & \\
Cefazolin & 7.2 & 10.9 \\
Cefuroxime & 10 & 7.8 \\
Cefoxitin & 56.4 & 73.4 \\
Ceftriaxone & 31.2 & 25 \\
Ceftazidime & 47.2 & 39.1 \\
Cefepime & 61.6 & 59.4 \\
Other $\beta$-lactams & & \\
Ampicillin & 0 & 0 \\
Aztreonam & 32.4 & 26.6 \\
Ticarcillin/clavulanate & 13.2 & 15.6 \\
Piperacillin/tazobactam & 55.6 & 57.8 \\
\hline
\end{tabular}




\begin{tabular}{|c|c|c|}
\hline Imipenem & 99.2 & 100 \\
\hline Meropenem & 99.6 & 100 \\
\hline \multicolumn{3}{|l|}{ Aminoglycosides } \\
\hline Amikacin & 72.8 & 71.4 \\
\hline Gentamicin & 43.2 & 42.2 \\
\hline Tobramycin & 30 & 20.6 \\
\hline \multicolumn{3}{|l|}{ Fluoroquinolones } \\
\hline Ciprofloxacin & 47.6 & 68.8 \\
\hline Levofloxacin & 49.2 & 71.9 \\
\hline Gatifloxacin & 50.4 & 73.4 \\
\hline Garenoxacin & 53.2 & 69.7 \\
\hline \multicolumn{3}{|l|}{ Others } \\
\hline Tetracycline & 31.6 & 40.6 \\
\hline $\begin{array}{l}\text { Trimethoprim/ } \\
\text { sulfamethoxazole }\end{array}$ & 27.3 & 26.6 \\
\hline \multicolumn{3}{|c|}{ Klebsiella pneumoniae (697/284) } \\
\hline \multicolumn{3}{|c|}{ Cephalosporins } \\
\hline Cefazolin & 1.6 & 1.4 \\
\hline Cefuroxime & 7 & 6 \\
\hline Cefoxitin & 72.7 & 77.1 \\
\hline Ceftriaxone & 18.4 & 14.8 \\
\hline Ceftazidime & 27.8 & 34.2 \\
\hline Cefepime & 48.4 & 43.7 \\
\hline \multicolumn{3}{|l|}{ Other $\beta$-lactams } \\
\hline Ampicillin & 0 & 0 \\
\hline Aztreonam & 17.6 & 14.4 \\
\hline Ticarcillin/clavulanate & 6.3 & 6 \\
\hline Piperacillin/tazobactam & 34.3 & 39.8 \\
\hline Imipenem & 99.6 & 100 \\
\hline Meropenem & 98.7 & 98.9 \\
\hline \multicolumn{3}{|l|}{ Aminoglycosides } \\
\hline Amikacin & 61.1 & 67.3 \\
\hline Gentamicin & 30.7 & 32.4 \\
\hline Tobramycin & 13.1 & 15.3 \\
\hline \multicolumn{3}{|l|}{ Fluoroquinolones } \\
\hline Ciprofloxacin & 75 & 80.3 \\
\hline Levofloxacin & 79.5 & 81.3 \\
\hline Gatifloxacin & 81.5 & 82.4 \\
\hline Garenoxacin & 76.6 & 73.7 \\
\hline \multicolumn{3}{|l|}{ Others } \\
\hline Tetracycline & 49 & 59.2 \\
\hline $\begin{array}{l}\text { Trimethoprim/ } \\
\text { sulfamethoxazole }\end{array}$ & 45.2 & 40.1 \\
\hline
\end{tabular}


Table 11d. Antimicrobial spectrum of drugs tested against ESBL-producing E. coli and K. pneumoniae isolated from January 1997 to December 2001 from patients with bacteremia and pneumonia

\begin{tabular}{|c|c|c|c|c|}
\hline \multirow{3}{*}{$\begin{array}{l}\text { Pathogen/ } \\
\text { Antimicrobial agent } \\
\text { E. coli }\end{array}$} & \multicolumn{4}{|c|}{ \% Susceptible strains (number of isolates tested) } \\
\hline & \multicolumn{2}{|c|}{ Latin America } & \multicolumn{2}{|c|}{ Brazil } \\
\hline & $\begin{array}{c}\text { Bacteremia } \\
(123)\end{array}$ & $\begin{array}{l}\text { Pneumonia } \\
\text { (30) }\end{array}$ & $\begin{array}{c}\text { Bacteremia } \\
\text { (28) }\end{array}$ & $\begin{array}{c}\text { Pneumonia } \\
\text { (11) }\end{array}$ \\
\hline \multicolumn{5}{|l|}{ Cephalosporins } \\
\hline Cefazolin & 8.1 & 0 & 10.7 & 0 \\
\hline Cefuroxime & 11.4 & 3.3 & 10.7 & 0 \\
\hline Cefoxitin & 60.2 & 73.3 & 75 & 100 \\
\hline Ceftriaxone & 30.1 & 10 & 17.9 & 0 \\
\hline Ceftazidime & 43.9 & 53.3 & 25 & 54.5 \\
\hline Cefepime & 63.4 & 43.3 & 57.1 & 45.5 \\
\hline \multicolumn{5}{|l|}{ Other $\beta$-lactams } \\
\hline Ampicillin & 0 & 0 & 0 & 0 \\
\hline Aztreonam & 33.3 & 10 & 21.4 & 18.2 \\
\hline Ticarcillin/clavulanate & 14.6 & 0 & 17.9 & 0 \\
\hline Piperacillin/tazobactam & 54.5 & 43.3 & 50 & 45.5 \\
\hline Imipenem & 100 & 96.7 & 100 & 100 \\
\hline Meropenem & 100 & 100 & 100 & 100 \\
\hline \multicolumn{5}{|l|}{ Aminoglycosides } \\
\hline Amikacin & 69.9 & 70 & 71.4 & 90.9 \\
\hline Gentamicin & 43.9 & 30 & 42.9 & 36.4 \\
\hline Tobramycin & 27.7 & 11.5 & 22.2 & 0 \\
\hline \multicolumn{5}{|l|}{ Fluoroquinolones } \\
\hline Ciprofloxacin & 52.8 & 56.7 & 71.4 & 90.9 \\
\hline Levofloxacin & 56.9 & 56.7 & 82.1 & 90.9 \\
\hline Gatifloxacin & 57.7 & 60 & 82.1 & 90.9 \\
\hline Garenoxacin & 60.3 & 47.1 & 73.3 & a \\
\hline \multicolumn{5}{|l|}{ Others } \\
\hline Tetracycline & 30.1 & 46.7 & 35.7 & 63.6 \\
\hline $\begin{array}{l}\text { Trimethoprim/ } \\
\text { sulfamethoxazole }\end{array}$ & 25.2 & 37.9 & 21.4 & 45.5 \\
\hline Klebsiella pneumoniae & $\begin{array}{c}\text { Bacteremia } \\
(405)\end{array}$ & $\begin{array}{c}\text { Pneumonia } \\
\text { (142) }\end{array}$ & $\begin{array}{c}\text { Bacteremia } \\
\text { (156) }\end{array}$ & $\begin{array}{c}\text { Pneumonia } \\
\qquad(65)\end{array}$ \\
\hline \multicolumn{5}{|l|}{ Cephalosporins } \\
\hline Cefazolin & 1 & 2.1 & 1.3 & 0 \\
\hline Cefuroxime & 8.1 & 2.8 & 6.4 & 0 \\
\hline Cefoxitin & 75.3 & 70.4 & 79.5 & 76.9 \\
\hline Ceftriaxone & 18.5 & 14.8 & 15.4 & 6.2 \\
\hline Ceftazidime & 27.9 & 24.6 & 37.2 & 18.5 \\
\hline
\end{tabular}




\begin{tabular}{lcccc}
\hline Cefepime & 49.6 & 47.9 & 44.9 & 47.7 \\
Other $\beta$-lactams & & & & \\
$\quad$ Ampicillin & 0 & 0 & 0 & 0 \\
Aztreonam & 17.5 & 15.5 & 16.7 & 7.7 \\
Ticarcillin/clavulanate & 7.2 & 6.3 & 7.7 & 3.1 \\
Piperacillin/tazobactam & 34.6 & 37.3 & 41.7 & 38.5 \\
Imipenem & 99.8 & 99.3 & 100 & 100 \\
Meropenem & 99.5 & 98.6 & 99.4 & 98.5 \\
Aminoglycosides & & & & \\
Amikacin & 59.3 & 67.6 & 64.1 & 33.8 \\
Gentamicin & 29.9 & 36.6 & 31.4 & 12.1 \\
Tobramycin & 12.2 & 14.8 & 15.6 & 80 \\
Fluoroquinolones & & & & 80 \\
Ciprofloxacin & 79.5 & 73.9 & 85.3 & 80 \\
Levofloxacin & 83.2 & 76.8 & 85.3 & 78.7 \\
Gatifloxacin & 84.9 & 76.1 & 87.2 & \\
Garenoxacin & 80.8 & 77.3 & 78.8 & 55.4 \\
Others & & & & 50 \\
Tetracycline & 49.8 & 54.2 & 63.5 & \\
Trimethoprim/ & & 52.5 & 34.6 & \\
sulfamethoxazole & 44.6 & & & \\
\hline
\end{tabular}

a. The results were not analyzed because the number of isolates was low $(<10)$.

resistance among pneumococci, are less frequently described in Latin America [1].

The main antimicrobial resistance problems the Latin American countries are presently facing are multidrug resistant (MDR) non-fermentative Gram-negative bacilli (Acinetobacter spp. and $P$. aeruginosa) and ESBL-prodcing Enterobacteriaceae [1,3,8,9,11-13]. Pseudomonas aeruginosa was the third and the fifth most frequently isolated pathogen in general and from blood stream infections, respectively. In addition, resistance rates were very high for all antimicrobial agents evaluated, except polymyxin B, which was evaluated since January 2001 only (Tables 2, 4, 6, and $8)$. The prevalence of isolates resistant to all antimicrobial agents except the polymyxins has been increasing continuously since the program started in 1997 [12]. Additionally, P. aeruginosa resistance rates were slightly higher among isolates collected in the Brazilian centers (Tables 2, 4, 6, and 8).
Acinetobacter spp. is much more prevalent and presented higher rates of antimicrobial resistance in Latin America, when compared to other regions evaluated by the SENTRY Program [1]. However, resistance rates to the carbapenems imipenem and meropenem were lower than those presented by $P$. aeruginosa (Tables 2, 4, 6, and 8). Besides these two carbapenems, other reasonable therapeutic options for empirical therapy of Acinetobacter spp. infections are the polymyxins and sulbactam. The SENTRY Program started evaluating these compounds in 2001 (polymyxin B) and 2002 (sulbactam), and the in vitro activity of these antimicrobial agents will be presented in future publications [14].

The rates of ESBL-producing Enterobacteriaceae, especially $K$. pneumoniae and E. coli, are among the highest in the world (Tables 2, 4, 6, 8, and 11) $[1,3,8,9,11]$ In addition to resistance to cephalosporins, broad-spectrum penicillins and 
monobactam, these isolates have also shown high rates of resistance to most antimicrobial agents, including aminoglycosides and fluoroquinolones. Although the fluoroquinolones remained active against some ESBL-producing K. pneumoniae (61.7\% to $87.7 \%$ susceptible), the carbapenems meropenem and imipenem remained the most reliable options for empirical therapy of infections due to ESBLproducing strains in the region (Table 11).

Other important resistance problems among Enterobacteriaceae include fluoroquinolone resistance due to altered target among E. coli [15]; and stably derepressed (AmpC) $\beta$-lactamases mediated resistance among Enterobacter cloacae, Citrobacter freundii, and other clinically important species. Ciprofloxacin susceptibility rates were as low as $85.5 \%$ among $E$. coli isolated from blood stream infections and $78.1 \%$ among isolates from patients hospitalized with lower respiratory tract infections (Tables 4 and 8).

Chromosomaly-inducible $\beta$-lactamases (AmpC or Bush group I) are produced by most Enterobacteriaceae species; however, some species, including E. cloacae, C. freundii, Serratia marcescens and indol-positive Proteus ("SPICE" or "CESP" group), can produce large amounts of these enzymes and have become resistant to third-generation cephalosporins, broad-spectrum penicillins and monobactams. In our study, E. cloacae resistance to ceftazidime, which indicates the production of the AmpC $\beta$-lactamase, was as high as $42.9 \%$ (Table 4 ). Cefepime ( $84.9-96.6 \%$ susceptibility) and the carbapenems imipenem and meropenem (92.9-100\% susceptibility) remained very active against $E$. cloacae and these other pathogens in the hospitals evaluated by the SENTRY program (Tables 2, 4, 6, and 8).

Among Gram-positive cocci, oxacillin resistance among staphylococci has remained an important problem in Latin America; however, rates varied significantly from hospital to hospital, even within a specific country. The vast majority of ORSA strains showed cross-resistance to most of the antimicrobial agents evaluated, except vancomycin, teicoplanin, quinupristin/dalfopristin and linezolid. Resistance to these agents was not detected among $S$. aureus isolates (only a small number of isolates had intermediateresistance to teicoplain or quinupristin/dalfopristin); however, decreased susceptibility to teicoplanin was relatively common among CoNS (90.3\% susceptibility; Table 9).

The prevalence of glycopeptide-resistant enterococci remained low in the Latin American hospitals evaluated by the SENTRY Program $(97.5 \%$ susceptibility in general) and its occurrence is usually linked to clonal dissemination of unique strains $[1,10,16]$.

The prevalence of penicillin-resistant pneumococci varied significantly among the Latin American countries evaluated, and it was especially low in Brazil (only 4.6\% with high-level resistance; Table 10). Macrolide resistance rates were around $10 \%$ to $12 \%$, with approximately half of the erythromycin resistant isolates showing cross-resistance to clindamycin MLS $_{B}$ phenotype). Tetracycline resistance rates were relatively elevated (around 20\%), while resistance rates to trimethoprim/sulfamethoxazole were very high, especially in Brazil (49.7\% susceptibility; Table 10).

Fluoroquinolone resistance was very rare among pneumococci (99.7\% susceptibility to gatifloxacin) and it was not detected among other streptococci species (Table 10). $\beta$-hemolytic streptococci remained very susceptible to penicillin and all other $\beta$-lactams evaluated. However, macrolide resistance was detected among the isolates of this pathogen (erythromycin susceptibility of $92.8 \%$ and $96.6 \%$ throughout Latin America and in Brazil, respectively). As expected, viridans group streptococci showed the highest rates of resistance among streptococci, with only $66.1 \%$ of the Latin American isolates being considered susceptible to penicillin.

Among the active surveillance systems, the SENTRY Program has incorporated extensive molecular strain typing and resistance genotyping as a means of providing additional information that may be useful for understanding pathogenic microorganisms worldwide $[1,17]$. In any surveillance program the phenotypic characterization of microorganisms and identification of epidemic clusters of certain species and resistance phenotypes is the primary role of the microbiology 
laboratory. In this way, the surveillance laboratory serves as an "early warning system", alerting the participating institutions of a potential problem with resistant organisms in a variety of patient populations [18].

The rapid emergence and dissemination of numerous drug resistances among bacteria has raised the necessity for controlling these pathogens in hospitals on a global scale; and the determination of clonality within a phenotypically identical resistant clusters will have a direct impact on the method of corrective intervention. On a broader scale, identification of resistant clones with extensive geographic range may provide insight into strain virulence and pathogenesis; and also may result in broader public health interventions, such as vaccination and antimicrobial restrictions aimed at limiting the spread of the pathogen and associated resistance problems [1].

Molecular methods may also be used to detect specific antimicrobial resistance genes (resistance genotyping) in a wide variety of organisms and when coupled with DNA fingerprinting have made substantial contributions to our understanding of the genetics of antimicrobial resistance and the spread of resistance determinants in the SENTRY Program medical centers $[7,15,19]$.

The main purpose of this report was to present a comprehensive tabular analysis of the antimicrobial susceptibility data of bacteria collected in the Latin American medical centers participating in the SENTRY Program. Antimicrobial resistances may vary significantly among Latin American countries and SENTRY Program evaluates only a small number of medical centers. However, the main objective of this program was to detect the most important problems of public health concern within the region and to assist/ guide regional intervention programs. The data presented here allow the identification of these principal resistance problems and the comparison of the in vitro activity and spectrum of a large number of antimicrobial agents not often tested in most hospitals. Lastly, we emphasize that empirical therapy should be guided by local susceptibility data when those are available, but in the absence of such information, surveillance data can help with therapeutic choices.

\section{Acknowledgements}

We express our appreciation to all medical technicians who have worked in SENTRY. The SENTRY Latin America Study Group in 1997-2001 includes: Helio S. Sader, Ana C. Gales (São Paulo, Brazil - Latin American Coordinator); Jorge Sampaio (Lâmina Laboratory, Rio de Janeiro, Brazil; Cássia Zoccoli (Santa Lúzia Laboratory, Florianopolis, Brazil); Afonso Barth (Hospital de Clinicas, Porto Alegre, Brazil); Julival Ribeiro (Hospital de Base, Brasília, Brazil); José M. Casellas (Centro de Estudios en Antimicrobianos, San Isidro, Argentina); Jorgelina Smayevsky (Microbiology Laboratory C.E.M.I.C., Buenos Aires, Argentina); Valeria Prado (Faculdad de Medicina de Chile, Santiago, Chile); Elizabeth Palavecino/ Patricia Garcia (Universidad Catolica del Chile, Santiago, Chile); Homero Bagnulo (Hospital Maciel, Montivideo, Uruguay); Jaime A. Robledo (Corporation para Investigaciones Biologicas, Medellin, Colombia); Jose Sifuentes-Osornio (Instituto Nacional de la Nutricion, Ciudade del Mexico, Mexico); and Manuel Guzmán-Blanco (Hospital Vargas, Caracas, Venezuela). Soraya Andrade, Rodrigo E. Mendes and Suzane Silbert provided excellent technical support. The SENTRY Antimicrobial Surveillance Program has been sponsored by a research/ educational grant from Bristol-Myers Squibb.

\section{References}

1. Jones R.N. [Ed.]. Global aspects of antimicrobial resistance among key bacterial pathogens. Results from the 19971999 SENTRY Antimicrobial Program. Clin Infect Dis 2001;32(suppl 2):S81-S156.

2. Pfaller M.A., Jones R.N., Doern G.V., et al. Survey of blood stream infections attributable to Gram-positive cocci: frequency of occurrence and antimicrobial susceptibility of isolates collected in 1997 in the United States, Canada, and Latin America from the SENTRY Antimicrobial Surveillance Program. Diag Microbiol Infect Dis 1999;33:283-97.

3. Sader H.S., Sampaio J.L.M., Zoccoli C., Jones R.N. Results of the SENTRY antimicrobial surveillance program results in three Brazilian medical centers for 1997. Braz J Infect Dis 1999;3:63-79. 
4. Sader H.S., Jones R.N., Gales A.C., et al. Antimicrobial susceptibility patterns for pathogens isolated from patients in Latin American medical centers with a diagnosis of pneumonia: Results from the SENTRY Antimicrobial Surveillance Program (1997). Diag Microbiol Infect Dis 1998;32:289-301.

5. National Committee for Clinical Laboratory Standards (NCCLS). Approved standard M7-A6: Methods for dilution antimicrobial susceptibility test for bacteria that grow aerobicaly. Sixth edition. Wayne, PA, 2003.

6. National Committee for Clinical Laboratory Standards (NCCLS). Performance standards for antimicrobial susceptibility testing; Thirteenth informational supplement. Document M100-S13. Wayne, PA, 2003.

7. Diekema D.J., Pfaller M.A., Turnidge J., et al. Genetic relatedness of multidrug-resistant, methicillin (oxacillin)resistant Staphylococcus aureus bloodstream isolates from SENTRY Antimicrobial Resistance Surveillance Centers worldwide, 1998. SENTRY Participants Group. Microb Drug Resist 2000;6:213-21.

8. Sader H.S., Jones R.N., Winokur P.L., et al. Antimicrobial susceptibility of bacteria causing urinary tract infections in Latin American hospitals: Results from the SENTRY Antimicrobial Surveillance Program (1997). Clin Microbiol Infect 1999;5:478-87.

9. Sader H.S., Pfaller M.A., Jones R.N., et al. Bacterial pathogens isolated from patients with blood stream infections in Latin America, 1997: Frequency of occurrence and antimicrobial susceptibility patterns from the SENTRY Antimicrobial Surveillance Program. Braz J Infect Dis 1999;3:97110.

10. Cereda R.F., Gales A.C., Silbert S., et al. Molecular typing and antimicrobial susceptibility of vancomycinresistant Enterococcus faecium (VRE) in Brazil. Infect Control Hosp Epidemiol 2002;23:19-22.

11. Sader H.S. Antimicrobial Resistance in Brazil: Comparison of results from two multicenter studies. Braz J Infect Dis 2000;4:91-9.

12. Andrade S.S., Jones R.N., Gales A.C., Sader H.S. Increasing Prevalence of Antimicrobial Resistance Among P. aeruginosa isolates in Latin America Medical Centers: 5-Year Report of the SENTRY Antimicrobial Surveillance Program (1997-2001). J Antimicrob Chemother 2003; $52: 140-1$.

13. Sader H.S., Jones R.N., Andrade-Baiocchi S., et al. Four-year evaluation of frequency of occurrence and antimicrobial susceptibility patterns of bacteria from blood stream infections in Latin American medical centers. Diagn Microbiol Infect Dis 2002; $44: 273-80$.
14. Gales A.C., Silbert S., Jones R.N., Sader H.S. Assessment of the antimicrobial activity of polymyxin B against contemporary Gram-negative bacilli isolated in Latin America: Results of SENTRY Antimicrobial Surveillance Program. In the abstract book of $42^{\text {nd }}$ Interscience Conference on Antimicrobial Agents and Chemotherapy, San Diego, CA, 2002.

15. Gales A.C., Gordon K.A., Wilke W.W., et al. Occurrence of single-point gyr A mutations among ciprofloxacinsusceptible Escherichia coli isolates causing urinary tract infections in Latin America. Diagn Microbiol Infect Dis 2000;36:61-4.

16. Cordeiro J.C.R., Silbert S., Reis A.O., Sader H.S. Interhospital dissemination of vancomycin-resistant Enterococcus faecalis in Brazil. Clinical Microbiology Infection 2004; 10:260-2.

17. Hollis R.J., Bruce J.L., Fritschel S.J., Pfaller M.A. Comparative evaluation of an automated ribotyping instrument versus pulsed-field gel electrophoresis for epidemiological investigation of clinical isolates of bacteria. Diagn Microbiol Infect Dis 1999;34:263-8.

18. Pfaller M.A., Herwaldt L.A. The clinical microbiology laboratory and infection control: Emerging pathogens, antimicrobial resistance and new technology. Clin Infect Dis 1997; $25: 858-70$.

19. Gales A.C., Jones R.N., Pfaller M.A., et al. Two-year assessment of the pathogen frequency and antimicrobial resistance patterns among organisms isolated from skin and soft tissue infections in Latin American hospitals: Results from the SENTRY Antimicrobial Surveillance Program, 1997-98. SENTRY Study Group. Emerg Infect Dis 2000;4:75-84. 